\title{
First South African Congress of Oncology 2019
}

This conference report encompasses abstracts and posters presented at the First South African Congress of Oncology on 'Transporting Cancer Care into 2020 and Beyond', 08-11 August 2019, at Cape Town International Convention Centre, Cape Town, South Africa. The National Congress is held in conjunction with the South African Society of Clinical and Radiation Oncology (SASCRO), the South African Children's Cancer Study Group (SACCSG), the South African Association of Physicists in Medicine and Biology (SAAPMB), the South African Society of Gynaecologic Oncology (SASGO), the National Oncology Nursing Society of South Africa (NONSA), the South African Oncology Social Workers' Forum (SAOSWF), the South African Society of Oncology Pharmacists (SASOP) and the Society of Radiation Therapists of South Africa (SRTSA).

\section{Investigation of potential skin toxicity for patients receiving breast IORT using EBT3 Gafchromic ${ }^{\circledR}$ film}

\section{M. van Heerden}

Netcare Medical Physics CoE, Netcare Ltd., Johannesburg, South Africa

Objectives: To accurately calibrate EBT3 Gafchromic ${ }^{\circledR}$ film in the low kilovoltage X-ray energy range and utilise it for dosimetry to estimate the dose for breast cancer patients receiving IORT.

Method: EBT3 Gafchromic ${ }^{\circledR}$ film calibration was performed by exposing sections of the film to increasing dose levels using a Carl Zeiss INTRABEAM ${ }^{\circledast} 50 \mathrm{kV}$ X-ray source in an INTRABEAM $^{\circledR}$ water phantom. The absorbed dose to water could be determined by using a PTW TN34013 soft X-ray ionisation chamber and calculated using an algorithm specified by Zeiss. The irradiated film sections were scanned $42 \mathrm{~h}$ later using an Epson V750 Pro transmission scanner in a 48-bit RGB mode and analysis was performed in the red colour channel using ImageJ. Once the calibration curve had been established, it was verified at various dose levels before implementation as a dosimeter during IORT cases.

During each breast IORT case, a lumpectomy is performed and one of the spherical treatment applicators (diameters ranging $1 \mathrm{~cm}-5 \mathrm{~cm}$ ) is placed into the cavity. The applicator is attached to the probe-like end of the X-ray source. A dose of $20 \mathrm{~Gy}$ is prescribed at $0 \mathrm{~mm}$ (at the surface of the applicator). During this study, a $1 \times 1 \mathrm{~cm}^{2}$ section of film was placed onto the outer skin surface of the breast at a region where the skinto-applicator distance is minimal. The film was kept sterile by enclosing it with $3 \mathrm{M}^{\mathrm{TM}}$ Tegaderm ${ }^{\mathrm{TM}}$ transparent wound dressing before placement onto the patient. The influence of the Tegaderm ${ }^{\mathrm{TM}}$ on the film measurement was also evaluated.

Results: Of 22 patients who underwent the procedure, seven received skin doses between $3 \mathrm{~Gy}$ and $5 \mathrm{~Gy}$, which could result in low grade 1 skin reactions: erythema and epilation. The majority of the patients (12) received skin doses in the range of $5 \mathrm{~Gy}-10 \mathrm{~Gy}$, which could lead to high grade 1 reactions, resulting in dry desquamation. Two patients measured in the 10 Gy - 15 Gy dose range, which could lead to grade 2 reactions - dry or possibly moist desquamation and one patient received a dose of $17.37 \mathrm{~Gy}$, which may result in blister formation (grade 3). The dose measured by the film could be verified within $3.3 \%$ and the Tegaderm ${ }^{\mathrm{TM}}$ had negligible influence on the measurements. Data collection for this study is ongoing.

Conclusion: EBT3 Gafchromic ${ }^{\circledR}$ film was found to be a suitable tool in determining the skin dose for breast IORT patients.

\section{Evolution in breast cancer knowledge and management in the last four decades}

\section{M.A. Coccia-Portugal}

\section{Private Practice, Pretoria, South Africa}

2650 BC: Breast cancer was first documented by Egyptian physicians. After more than 45 centuries, the modern management of this disease was developed. In the last four decades, there has been a dramatic change in the knowledge of breast cancer biology, together with improvements in surgery, radiotherapy and medical therapies - thus impacting a change in its management. Until the 1970s local therapy was the only treatment and breast cancer was then recognised primarily as a local-regional disease.

1970s: First clinical trials using adjuvant systemic therapy for positive axillary glands (L-PAM and CMF) post-extensive surgery (mastectomy and axillary dissection). Primary systemic therapy for inoperable disease was initiated. Hormonal receptors were identified.

1980s: Adjuvant systemic therapy for negative axillary glands. The first target therapy (tamoxifen), anthracyclins, platinum and other medical therapies were further developed. Ongoing trials using breast conservative therapy and/or mastectomy, sentinel node biopsy and different systemic combination therapies for early disease.

1987: Breast cancer presented as a spectrum of diseases from 'local' to systemic when first detected.

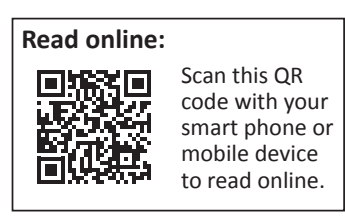

Dates: Published: 05 Aug. 2019

How to cite this article: Anon. First South African Congress of Oncology 2019. S.Afr. j. oncol. 2019;3(0), a90. https://doi.org/10.4102/sajo. v3i0.90

Copyright: @ 2019. The Authors. Licensee: AOSIS. This work is licensed under the Creative Commons Attribution License. 
The importance of local and systemic therapy was emphasised at diagnosis.

1990s: Taxanes, steroidal and non-steroidal aromatase inhibitors targeting hormonal receptor positive tumours and Trastuzumab for HER2Neu+ tumours used then only in metastatic disease.

\section{4: BRCA1 identification.}

1998: Review of all ongoing phase III clinical trials for $>20$ years comparing mastectomy versus conservative surgery. It was concluded that mastectomy is no longer the standard of care. The intensity of local treatment does not change patient survival. NSABP: Primary surgery versus primary chemotherapy showed no survival difference after 16 years of follow-up.

2000s: Gene expression patterns of breast cancer distinguishing tumour sub-types with clinical implications (Luminal A, Luminal B, HER2+, Triple Neg), thus diminishing the use of TNM anatomical classification. HER2 target therapy was used for early breast cancer and development of intraoperative radiotherapy, concluding that treatment must be tailored to patient characteristics, tumour stage and molecular profile.

2010s: The use of primary systemic therapy to evaluate tumour response at diagnosis, and the use of pathological response as a prognostic factor. PCR is associated with a prediction of long-term disease outcomes.

Conclusion for 2020s: Goal of improving the quantity and quality of life of breast cancer patients in developing countries. Breast cancer centres should be established in the private and government or academic centres where patient therapy can be individualised by a multidisciplinary team (radiology, pathology, medical oncology, surgery and radiotherapy) at diagnosis.

\section{Human cytolytic fusion proteins targeting chondroitin-sulphate proteoglycan 4 for the treatment of triple-negative breast cancer}

\section{N. Mungra ${ }^{1}$, S. Jordaan ${ }^{1}$, S. Chetty ${ }^{2}$ and S. Barth ${ }^{2}$}

${ }^{1}$ University of Cape Town, Cape Town, South Africa

${ }^{2}$ University of the Witwatersrand, Johannesburg, South Africa

Background: Conventional chemotherapeutic approaches face obstacles such as off-target toxicity and therapy resistance in certain cancers. Human cytolytic fusion proteins (hCFPs) are targeted therapeutic agents composed of a disease-specific humanised antibody-based ligand recombinantly fused with a conditionally apoptosis-inducing human oligopeptide. They exhibit low off-target toxicity and can overcome tumour resistance depending on the biomarkers selected. We generated hCFPs targeting chondroitin-sulphate proteoglycan (CSPG4), a biomarker of aggressive and treatment-resistant cancers, for the treatment of triple-negative breast cancer (TNBC). These hCFPs are designed to bind to CSPG4-positive TNBC cells and deliver cytotoxic proteins intracellularly, where they induce tumour cell death by disrupting biosynthesis (angiogenin) or mitosis (microtubule-associated protein tau or MAP tau) in a compartment-dependent manner.

Method: We designed recombinant hCFPs based on angiogenin and MAP tau as lead agents, as well as a CSPG4-targeting SNAP-tag fusion protein (as a fluorescent mimic for binding). All fusion proteins were expressed in a transient secretory mammalian expression system and purified from the cell culture supernatant by ion metal affinity chromatography. Binding of CSPG4 (scFv)-SNAP to CSPG4-positive and CSPG4negative TNBC cell lines was validated both quantitatively (by flow cytometry) and qualitatively (by confocal microscopy).

Results: Preliminary proof-of-concept data demonstrate that the CSPG4-targeting, fluorescently labelled CSPG4(scFv)SNAP binds effectively to CSPG4-positive TNBC cells.

Conclusion: The combination of hCFPs with a robust companion diagnostic tool such as SNAP-tag technology represents the first step towards the effective management of African TNBC.

\section{Linac-based stereotactic radiosurgery: Plan optimisation holds the key to quality}

\section{W. Shaw}

\section{University of the Free State, Bloemfontein, South Africa}

Objectives: This study compares five different linear accelerators in terms of their ability to perform highly conformal Linac-based stereotactic radiosurgery (LBSRS) for small-, medium- and large-sized brain lesions. Resultant dose distributions following rigorous plan optimisation are compared with GammaKnife (GK) dose distributions.

Method: Linac-based stereotactic radiosurgery is a wellestablished therapy for the treatment of arteriovenous malformations (AVM) and vestibular schwannomas (VS). Although the GK system has long been deemed the standard of care for cranial stereotactic irradiation, frameless imageguided radiosurgery allows more treatment plan optimisation flexibility and is offered on many linac platforms. LBSRS treatment plans were produced with ultra-modern volumetric modulated arc therapy techniques for small (VS and AVM), medium (AVM) and large (AVM) brain lesions on the hyperion treatment planning system. Lesions ranged from $0.5 \mathrm{cc}$ to $14.6 \mathrm{cc}$ in volume. Treatment plans were developed using beam models of five different linear accelerators: Varian TrueBeam with a millennium and high-definition collimating system; Elekta Synergy with an agility and apex collimating system, as well as an Elekta Beam Modulator, each with their unique collimating characteristics. A comparison was made between the final optimised dose distributions for the five linacs and compared with GK-planned dose distributions.

Results: Treatment plans for each of the linear accelerators in the study produced dose distributions that were acceptable for treatment. These included conformity and heterogeneity indices. Differences between the collimating systems and their resultant dose distributions were negligible for all target lesion volumes, except for the $0.5 \mathrm{cc}$ lesion that had slightly more 
favourable target volume conformity and OAR sparing with higher resolution collimation. However, higher resolution collimators performed only slightly better than those with courser collimation in this case. As expected, GK distributions resulted in a slightly better normal brain sparing at the cost of worse target dose uniformity and very long treatment times. The results indicate that any of these systems can deliver highly conformal treatments with little difference in dose distributions. Optimisation parameters, such as segment smoothing options, had a siginificanat impact on the final dose distributions than the differences in collimating systems.

Conclusion: Treatment plans for linear accelerators with modern collimation devices compare favourably with GK SRS treatment plans. Differences between linac collimation systems only become observable when target volumes are very small, typically $<0.5 \mathrm{cc}$. Planner experience and optimisation tools have a much bigger dosimetric impact on the treatment plan quality than the collimation systems.

\section{Carboplatin-based chemotherapy delivers superior results in children and adolescents with extracranial germ cell tumours: First report by the South African Children's Cancer Study Group, 1990-2015}

M. Hendricks ${ }^{1}$, A. Cois ${ }^{2,3}$, J. Geel ${ }^{4}$, J. du Plessis ${ }^{5}$, M. Bassingthwaighte ${ }^{6}$, G. Naidu ${ }^{6}$, B. Rowe ${ }^{6}$, A. Buchner ${ }^{7}$, F. Omar ${ }^{7}$, K. Thomas ${ }^{8}$, R. Uys ${ }^{9}$, A. van Zyl ${ }^{9}$, J. van Heerden $^{10,11}$, N. Mahlachana ${ }^{6}$, J. Vermeulen, A. Davidson ${ }^{1}$, L. Frazier ${ }^{13}$, K. Donald ${ }^{14}$ and M. Kruger ${ }^{7}$

${ }^{1}$ Haematology Oncology Service, Department of Paediatrics and Child Health, Red Cross War Memorial Children's Hospital, University of Cape Town, Cape Town, South Africa

${ }^{2}$ Burden of Disease Research Unit, South African Medical Research Council, Cape Town, South Africa

${ }^{3}$ Division of Epidemiology and Biostatistics, School of Public Health and Family Medicine, University of Cape Town, Cape Town, South Africa ${ }^{4}$ Division of Paediatric Haematology Oncology, Department of Paediatrics and Child Health, Charlotte Maxeke Johannesburg Academic Hospital, University of the Witwatersrand, Johannesburg, South Africa

${ }^{5}$ Division of Paediatric Haematology Oncology, Department of Paediatrics, Universitas Hospital, University of the Free State, Bloemfontein, South Africa

${ }^{6}$ Division of Paediatric Haematology Oncology, Chris Hani Baragwanath Academic Hospital, University of the Witwatersrand, Soweto, South Africa

${ }^{7}$ Paediatric Haematology Oncology, Department of Paediatrics, Steve Biko Academic Hospital, University of Pretoria, Tshwane, South Africa ${ }^{8}$ Paediatric Haematology Oncology, Department of Paediatrics and Child Health, Frere Hospital, East London, South Africa

${ }^{9}$ Paediatric Haematology Oncology, Department of Paediatrics and Child Health, Tygerberg Hospital, Stellenbosch University, Cape Town, South Africa

${ }^{10}$ Pediatric Haematology Oncology, Department of Paediatrics and Child Health, Pietermaritzburg Metro Complex, University of KwaZulu-Natal, Pietermaritzburg, South Africa

${ }^{11}$ Paediatric Haematology and Oncology, Department of

Paediatrics and Child Health, University of Antwerp, Antwerp University Hospital, Antwerp, Belgium

${ }^{12}$ Paediatric Haematology Oncology, Department of Paediatrics and Child Health, Port Elizabeth Provincial Hospital, Walter Sisulu University, Port Elizabeth, South Africa
${ }^{13}$ Peediatric Oncology, Dana-Farber/Boston Children's Cancer and Blood Disorders Center, Harvard University, Boston, MA, United States of America

${ }^{14}$ Division of Neurodevelopment, Department of Paediatrics and Child Health, Red Cross War Memorial Children's Hospital, University of Cape Town, Cape Town, South Africa

Background: Malignant extracranial germ cell tumours (MEGCTs) require a comprehensive multidisciplinary team approach to achieve good survival rates and good quality of survival. In preparation for harmonised national treatment protocol development, a national retrospective analysis was performed to evaluate treatment outcomes for children and adolescents with MEGCTs.

Methods: Retrospective data from nine South African paediatric oncology units were analysed to determine 5-year overall survival (OS) and prognostic factors. Carboplatin, etoposide and bleomycin (CEB) and bleomycin, etoposide and carboplatin (BEP) were most frequently used and longterm toxicities are described.

Results: A total of 217 children and adolescents (64 men, median age 25.67 months; 153 women, median age 98.33 months) were diagnosed with MEGCTs. Disease stage significantly affected the 5-year OS: stage $1(95.9 \%)$, stage $2(94.3 \%)$, stage $3(75.2 \%)$ and stage $4(59.8 \%)$ ( $p=0.00002$, confidence interval [CI]: 95\%). The use of protocol also impacted the outcome: Jeb $(87.6 \%)$, BEP $(80.5 \%)$, PEB $(66.5 \%)$ and others $(49.6 \%)(p=0.002)$. Only 6/82 (7.3\%) patients who received cisplatin-based chemotherapy had a documented glomerular filtration rate at diagnosis and only $10 / 82(12.2 \%)$ had an audiogram. Thirteen patients receiving cisplatin-based chemotherapy developed hearing deficits, of whom four were severe. Causes of death in 36 patients included disease progression (30), infection (3), pulmonary fibrosis (1), secondary acute myeloid leukaemia (1) and surgical complications (1).

Conclusions: In the largest cohort of MEGCTs from subSaharan Africa, we demonstrated a 5-year OS of $80.3 \%$, with stage 4 patients having only achieved a 59.9\% OS. Protocol use impacted survival, with JEb showing clear superiority. Of concern, GFR and audiogram assessment for prechemotherapy was performed in a minority of patients. Development and implementation of a standardised national protocol is intended to improve the survival rate, especially for those with advanced disease, and will standardise the management of patients with long-term sequelae.

\section{The management of neuroblastoma in South Africa between 2000 and 2014}

J. Van Heerden ${ }^{1,2}$, M. Hendricks ${ }^{3}$, J. Geel ${ }^{4}$, B. Sartorius ${ }^{5,6}$, J. Du Plessis ${ }^{7}$, A. Büchner ${ }^{8}$, G.P. Hadley ${ }^{5}$, B. van Emmenes ${ }^{9}$, G. Naidu ${ }^{4}$, A. van $Z \mathrm{yl}^{1}$ and M. Kruger ${ }^{1}$

${ }^{1}$ Stellenbosch University, Stellenbosch, South Africa ${ }^{2}$ Paediatric Haematology and Oncology, Department of Paediatrics and Child Health, University of Antwerp, Antwerp University Hospital, Antwerp, Belgium 
${ }^{3}$ Haematology Oncology Service, Department of Paediatrics and Child Health, Red Cross War Memorial Children's Hospital, University of Cape Town, Cape Town, South Africa ${ }^{4}$ Division of Paediatric Haematology Oncology, Department of Paediatrics and Child Health, Charlotte Maxeke Johannesburg Academic Hospital, University of the Witwatersrand, Johannesburg, South Africa

${ }^{5}$ Public Health Medicine, School of Nursing and Public Health, College of Health Sciences, Nelson R Mandela School of Medicine, University of KwaZulu-Natal, Durban, South Africa

${ }^{6}$ Institute for Health Metrics and Evaluation, University of Washington, Seattle, WA, United States of America

${ }^{7}$ University of the Free State, Bloemfontein, South Africa

${ }^{8}$ University of Pretoria, Pretoria, South Africa

${ }^{9}$ Frere Hospital, East London, South Africa

Background: Neuroblastoma (NB) in low- and middle-income countries (LMIC) has a poor outcome compared to highincome countries (HIC).

Objectives: A multi-institutional retrospective study across South Africa (SA) was conducted to determine outcomes.

Methods: Patients treated between January 2000 and December 2014 in nine paediatric oncology units (POUs) were included. Demographic data, disease stage, treatment protocol and outcome were collected after custodian consent and individual consent waivers. Risk stratification was redetermined. Kaplan-Meier curves and Cox regression models were used for data analysis, metastatic post-induction remission and 2-year overall survival (OS).

Results: Data from 390 patients with neuroblastoma were included. The prevalence was 3.1/1 000000 for 2014, representing $6.3 \%$ of all reported childhood malignancies. The median age was 39.9 months (standard deviation (s.d.) 36.8 , range $0-201$ months). The majority of patients presented with stage 4 disease $(70 \%)$. The main chemotherapy regimens were OPEC/OJEC (44.8\%), St Jude NB84 protocol (28.96\%) and Rapid COJEC (22.17\%). Only 44.4\% received surgical interventions across all risk groups, while $16.5 \%$ of high-risk (HR) patients received radiotherapy. The 2-year OS was $94.1 \%$ for very low risk, $81.6 \%$ for low risk, $66.7 \%$ for intermediate risk and $27.6 \%$ for HR groups $(p<0.001,95 \%$ $\mathrm{CI})$. The 2 -year OS of the NB84 was $41.0 \%$ in comparison to OPEC/OJEC with $32.5 \%$ and Rapid COJEC with $24.8 \%$ ( $p=0.039,95 \% \mathrm{CI})$. OPEC/OJEC had the least treatmentrelated deaths $(2.0 \%)$, followed by NB84 (6.2\%) and Rapid COJEC $(6.3 \%)(p=0.037,95 \% \mathrm{CI})$. Median survival time for the whole group was 13.0 months (mean 41.9 months, range $0.1-209$ months). On multivariate analysis, stage $(p<0.0001$, $65 \% \mathrm{CI}), \mathrm{LDH}>750 \mathrm{U} / \mathrm{L}(p<0.0001,95 \% \mathrm{CI})$, ferritin $>$ $210 \mathrm{~g} / \mathrm{dL}(p<0.02,95 \% \mathrm{CI}), \mathrm{MYCN}$ amplification $(p=0.01$, $95 \% \mathrm{CI})$, risk classification $(p<0.0001,95 \% \mathrm{CI})$, chemotherapy regimens $(p<0.04,95 \% \mathrm{CI})$ and surgical resection $(p<0.01$, $95 \% \mathrm{CI}$ ) were identified as significant prognostic factors.

Conclusion: Limited disease had comparable OS to HIC. Poor OS was related to advanced disease. South Africa should investigate reasons for advanced disease and harmonise treatment by implementing a standard national treatment protocol.
Favourable outcomes for South African children and adolescents with mature and immature teratomas (1990-2015): First report by the South African Children's Cancer Study Group

M. Hendricks ${ }^{1}$, A. Cois ${ }^{2,3}$, J. du Plessis ${ }^{4}$, J. Geel ${ }^{5}$, M. Bassingthwaighte ${ }^{6}$, G. Naidu ${ }^{6}$, B. Rowe ${ }^{6}$, A. van Zyl7, R. Uys ${ }^{7}$, J. van Heerden ${ }^{8,9}$, K. Thomas ${ }^{10}$, A. Buchner ${ }^{11}$, F. Omar ${ }^{11}$, N. Mahlachana ${ }^{6}$, J. Vermeulen ${ }^{12}$, A. Davidson ${ }^{1}$, L. Frazier ${ }^{13}$, K. Donald ${ }^{14}$ and M. Kruger ${ }^{7}$

${ }^{1}$ Haematology Oncology Service, Department of Paediatrics and Child Health, Red Cross War Memorial Children's Hospital, University of Cape Town, Cape Town, South Africa

${ }^{2}$ Burden of Disease Research Unit, South African Medical Research Council, Cape Town, South Africa

${ }^{3}$ Division of Epidemiology and Biostatistics, School of Public Health and Family Medicine, University of Cape Town, Cape Town, South Africa

${ }^{4}$ Division of Paediatric Haematology Oncology, Department of Paediatrics, Universitas Hospital, University of the Free State, Bloemfontein, South Africa

${ }^{5}$ Division of Paediatric Haematology Oncology, Department of Paediatrics and Child Health, Charlotte Maxeke Johannesburg Academic Hospital, University of the Witwatersrand, Johannesburg, South Africa

${ }^{6}$ Division of Paediatric Haematology Oncology, Chris Hani Baragwanath Academic Hospital, University of the Witwatersrand, Soweto, South Africa

${ }^{7}$ Paediatric Haematology Oncology, Department of Paediatrics and Child Health, Tygerberg Hospital, Stellenbosch University, Cape Town, South Africa

${ }^{8}$ Pediatric Haematology Oncology, Department of Paediatrics and Child Health, Pietermaritzburg Metropolitan Hospital Complex, University of KwaZulu-Natal, Pietermaritzburg, South Africa ${ }^{9}$ Paediatric Haematology and Oncology, Department of Paediatrics and Child Health, Antwerp University Hospital, University of Antwerp, Antwerp, Belgium

${ }^{10}$ Paedaitric Haematology Oncology, Department of Paediatrics and Child Health, Frere Hospital, East London, South Africa ${ }^{11}$ Paediatric Haematology Oncology, Department of Paediatrics, Steve Biko Academic Hospital, University of Pretoria, Tshwane, South Africa

${ }^{12}$ Paediatric Haematology Oncology, Department of Paediatrics and Child Health, Port Elizabeth Provincial Hospital, Walter Sisulu University, Port Elizabeth, South Africa

${ }^{13}$ Pediatric Oncology, Dana-Farber/Boston Children's Cancer and Blood Disorders Center, Harvard University, Boston, MA, United States of America

${ }^{14}$ Division of Neurodevelopment, Department of Paediatrics and Child Health, Red Cross War Memorial Children's Hospital, University of Cape Town, Cape Town, South Africa

Background: Outcomes of rare paediatric teratomas have not previously been reported, and treatment regimens have not yet been standardised in the middle income setting.

Objectives: To evaluate treatment outcomes for children and adolescents with biopsy-proven mature and immature teratomas in preparation for harmonisation of management approaches.

Methods: Retrospective data by folder review were collated from nine South African paediatric oncology units. 
Kaplan-Meier analysis with Cox regression was performed to determine the 5-year overall survival (OS) and prognostic factors.

Results: From January 1990 to December 2015, 64 patients (15 men, median age 3.47 months; 47 women, median age 10.83 months) were diagnosed with mature teratomas and 84 patients ( 20 men, median 0.85 months; 64 women, median 30.02 months) were diagnosed with immature teratomas. Definitive primary resection was performed in $58 / 62$ patients with mature teratomas. In patients with immature teratomas, $62 / 84$ had primary surgery, which was definitive in 53.3\% $(33 / 62)$ cases. The 5-year OS for patients with mature teratomas was $92.5 \%$ and it was $80 \%$ for those with immature teratomas. For the combined group, outcome was significantly influenced by disease stage: stage $1(97.3 \%)$, stage $2(100 \%)$, stage $3(64.4 \%)$ and stage $4(55.5 \%)(p=0.00001)$. Five patients with mature teratomas died: two from post-operative complications, one from disease, one from infection and one owing to unrelated reason. Fourteen patients with immature teratomas died: 11 from disease progression, 2 from unrelated reasons and 1 from infection. Loss-to-follow-up was reported in 21/62 (38\%) patients with mature teratomas and $11 / 8(13.1 \%)$ patients with immature teratomas. All had stage 1 disease and they underwent surgery alone.

Conclusions: Five-year OS for the entire group was $84.7 \%$, with poor outcomes for stage 4 disease. This is the largest cohort of its kind reported in sub-Saharan Africa. Harmonisation of a national protocol will assist in standardising surgical approaches, indications for chemotherapy and follow-up guidelines and is intended to improve prognosis.

\section{Favourable outcomes in children and adolescents with sex cord stromal tumours (1990-2015): First report by the South African Children's Cancer Study Group}

M. Hendricks ${ }^{1}$, A. Cois ${ }^{2,3}$, J. du Plessis ${ }^{4}$, M. Bassingthwaighte ${ }^{5}$, G. Naidu ${ }^{5}$, A. van Zyl ${ }^{6}$, J. van Heerden ${ }^{7,8}$, R. Uys ${ }^{6}$, A. Buchner ${ }^{9}$, B. Rowe ${ }^{5}$, F. Omar ${ }^{9}$, J. Geel ${ }^{10}$, N. Mahlachana ${ }^{5}$, K. Thomas ${ }^{11}$, J. Vermeulen ${ }^{12}$, A. Davidson ${ }^{1}$, A.L. Frazier $^{13}$, K. Donald ${ }^{14}$ and M. Kruger

${ }^{1}$ Haematology Oncology Service, Department of Paediatrics and Child Health, Red Cross War Memorial Children's Hospital, University of Cape Town, Cape Town, South Africa

${ }^{2}$ Burden of Disease Research Unit, South African Medical Research Council, Cape Town, South Africa

${ }^{3}$ Division of Epidemiology and Biostatistics, School of Public Health and Family Medicine, University of Cape Town, Cape Town, South Africa

${ }^{4}$ Division of Paediatric Haematology Oncology, Department of Paediatrics, Universitas Hospital, University of the Free State, Bloemfontein, South Africa

${ }^{5}$ Division of Paediatric Haematology Oncology, Chris Hani Baragwanath Academic Hospital, University of the Witwatersrand, Soweto, Gauteng

${ }^{6}$ Paediatric Haematology Oncology, Department of Paediatrics and Child Health, Tygerberg Hospital, Stellenbosch University, Cape Town, South Africa
${ }^{7}$ Paediatric Haematology Oncology, Department of Paediatrics and Child Health, Pietermaritzburg Metropolitan Hospital Complex, University of KwaZulu-Natal, Pietermaritzburg, South Africa ${ }^{8}$ Paediatric Haematology and Oncology, Department of Paediatrics and Child Health, University of Antwerp, Antwerp University Hospital, Antwerp, Belgium

${ }^{9}$ Paediatric Haematology Oncology, Department of Paediatrics, Steve Biko Academic Hospital, University of Pretoria, Tshwane, South Africa

${ }^{10}$ Division of Paediatric Haematology Oncology, Department of Paediatrics and Child Health, Charlotte Maxeke Johannesburg Academic Hospital, University of the Witwatersrand, Johannesburg, South Africa

${ }^{11}$ Paedaitric Haematology Oncology, Department of Paediatrics and Child Health, Frere Hospital, East London, South Africa

${ }^{12}$ Paediatric Haematology Oncology, Department of Paediatrics and Child Health, Port Elizabeth Provincial Hospital, Walter Sisulu University, Port Elizabeth, South Africa

${ }^{13}$ Pediatric Oncology, Dana-Farber/Boston Children's Cancer and Blood Disorders Center, Harvard University, Boston, MA, United States of America

${ }^{14}$ Neurodevelopmental Service, Department of Paediatrics and Child Health, Red Cross War Memorial Children's Hospital, University of Cape Town, Cape Town, South Africa

Background: Paediatric sex cord stromal tumours (SCSTs) are extremely rare and there is no data on outcomes reported from Africa.

Objectives: To evaluate treatment outcomes for children and adolescents with biopsy-proven SCSTs in preparation for the introduction of a national protocol.

Methods: Retrospective data were collated from nine South African paediatric oncology units. Kaplan-Meier analysis with Cox regression was performed to determine the 5-year overall survival (OS)

Results: From January 1990 to December 2015, 24 patients (4 men, median age 100.52 months; 20 women, median age 78.18 months) were diagnosed with SCSTs. Patient histologies included 1 thecoma, 12 Sertoli-Leydig cell tumours and 11 juvenile granulosa cell tumours. Only disease stage impacted the OS: stage $1(100 \%)$, stage $2(0 \%)$, stage $3(80 \%)$ and stage 4 (50\%). Twenty-one patients underwent primary surgery and the remaining three followed neo-adjuvant chemotherapy. Patients identified with FIGO stage 1C disease and above received chemotherapy, except for one patient with FIGO stage 3A disease, who is a survivor. One patient with FIGO stage 1A disease received chemotherapy for rising markers and survived. Twelve patients received chemotherapy: six carboplatin, etoposide and bleomycin (JEB), four bleomycin, etoposide and cisplatin (BEP) and two others. One patient who received JEB was changed to cisplatin, etoposide, bleomycin (PEB) after two courses. Two patients developed hearing impairment: one received BEP and the other PEB. Only three patients had audiograms and two had glomerular filtration rate (GFR) testing prior to chemotherapy. Three patients died of disease. There were no treatment-related deaths.

Conclusions: Although the numbers in this cohort are small, this study represents a relatively large cohort and the OS of 
$82.6 \%$ is encouraging. It is essential that the management of rare tumours such as SCSTs should be standardised to ensure the best possible OS and to limit long-term sequelae. Based on these results, a standardised management protocol has been designed.

\section{Paediatric solid tumours in low- to middle-income countries: A decade of experience from a South African hospital}

\section{S. Mushunje, D.S. Harrison and G. Naidu}

\section{University of the Witwatersrand, Johannesburg, South Africa}

Background: There are few modifiable risk factors for solid tumours in children, except in the presence of a predisposing condition or syndrome; therefore, early diagnosis and treatment are important in improving survival. Reporting the incidence, demographic data, presentation and outcomes of these solid tumours may improve the awareness and allocation of resources for their management. There is paucity of literature on the incidence, prevalence, demographics and treatment of paediatric solid tumours in low- to middleincome countries (LMICs) including South Africa.

Objectives: The aim of this study was to present, describe and interpret the disease burden, demographics and stages at presentation of paediatric solid tumours at Chris Hani Baragwanath Academic Hospital (CHBAH).

Methods: A retrospective review of medical records of children below 16 years with solid tumours treated at CHBAH from 01 January 2007 to 31 December 2016 was carried out. Central nervous system, eye, bone and medically managed tumours were excluded. Data on the total number of all paediatric cancers seen each year at the Oncology Department were obtained for comparison.

Results: There were 387 solid tumours, accounting for $33 \%$ of all paediatric oncology cases. Of these tumours, 85\% were malignant and only 3\% occurred in children with a predisposing condition. Nephroblastoma was the most common tumour, accounting for $28 \%$, followed by rhabdomyosarcoma at $15.5 \%$, neuroblastoma at $12.5 \%$, germ cell tumours at $12 \%$ and hepatoblastoma at $4 \%$. Peaks in occurrence were seen in nephroblastoma, rhabdomyosarcoma and neuroblastoma among 1-5 years age group, with $48 \%$ of all the tumours studied occurring within this age group. Late presentation was common, with $52 \%$ presenting with advanced disease. Over the 10-year period, 296 oncological surgeries were performed, averaging 30 operations per year, including surgeries performed for lung metastases.

Conclusion: The solid tumours studied accounted for onethird of all oncology cases encountered in children at $\mathrm{CHBAH}$, with over $50 \%$ of the malignant tumours presenting late with advanced disease. Programmes put in place to create awareness on signs and symptoms of paediatric solid tumours encourage that early presentation, early referral and prompt management will improve survival outcome in paediatric solid tumours at $\mathrm{CHBAH}$. These programmes may be targeted towards the 1-5 years age group, among whom half of these solid tumours occur.

\section{Setting up a new radiotherapy facility in Kenya: The Eldoret experience}

\section{P.K. Alusa ${ }^{1}$ and G.J.J. Korf ${ }^{1,2}$}

${ }^{1}$ Eldoret Comprehensive Cancer Center - Equra Health Kenya, Eldoret, Kenya

${ }^{2}$ Equra Health Africa, Stellenbosch University, Stellenbosch, South Africa

Background: According to the Ministry of Health, Kenya, cancer is the third leading cause of death after infectious and cardiovascular diseases. Literature reports that in 2012 there was an estimated 37000 new cancer cases and 28000 cancerrelated deaths in Kenya. Approximately 15\% of the global cancer burden is attributable to infectious agents, with higher percentages in developing countries. In Kenya, as in common in many African countries, cancer patients have to travel a long distance (as far as $600 \mathrm{~km}$ away) to access treatment. There were only one public facility and five private facilities, all based in the capital city of Nairobi, when Equra Health Kenya decided to broaden easier access to cancer care services in other parts of Kenya. Eldoret, one of the new growing cities in the country, is located $500 \mathrm{~km}$ west from Nairobi. Access to radiotherapy services especially in Kenya remains a challenge. The situation in the public sector is even more challenging, as well as in the private sector where many patients, when commencing radiation therapy, are at advanced stage of their disease.

Method: The cancer centre in Eldoret was planned, constructed and operationalised as a total new facility and this required the project to be tackled in various phases:

- Initial stages: business feasibility studies, identifying local clinical partners, site identification, tendering for construction and construction of the facility

- Equipping and staffing: equipment identification and ordering, delivery and installation, acceptance, commissioning and the recruitment of staff

- Operationalising of the facility: staff orientation, protocols and policies implementation, safe work procedures and accreditation of the facility.

Results: Various challenges occurred along the journey. These related to, for example, the initial and ongoing importation process of equipment, parts and radioactive sources, different construction methods, unreliable electricity supply, staff availability and centre accreditation challenges. Despite the challenges, the impact of opening a cancer treatment facility outside Nairobi has been significant. The number of radiotherapy and brachytherapy patients increased dramatically over the course of 24 months, resulting in reduced travelling costs and improved cancer support closer to where people live and work.

Conclusion: With the successful launch of the modern chemotherapy and radiotherapy facility in Eldoret, cancer 
patients no longer have to experience the burden of traveling more than $600 \mathrm{~km}$ to access similar facilities in the capital city of Nairobi. Because of local access to necessary cancer treatment options, the waiting time from diagnosis to treatment has greatly reduced for a large number of patients.

\section{Demonstration of a three-dimensional graphics, interactive radiotherapy simulator for training and teaching}

\section{J.J. Botha}

\section{Groote Schuur Hospital, Cape Town, South Africa}

Background: Familiarity and confidence are important aspects in ensuring that equipment are used properly, with care and to the full ability of the equipment. It is ideal to have a treatment unit available on which staff can learn and practise; however, because of the high cost of treatment machines and the need to use the treatment units optimally for patient treatments, it is not possible or feasible to have treatment units ideal for staff training. To overcome this problem, an interactive, computer simulation is needed.

Objectives: The aim of this project was to develop a computer simulator for radiotherapy, which will address all aspects of radiotherapy, from basic equipment use to patient setup, as well as image production.

Method: The first step was to determine what aspects were required to be simulated in terms of detail and complexity. The next step was to design three-dimensional (3D) models of the selected treatment unit objects. These models were then created using 3D modelling software. These 3D models are then combined and a computer program was written to manipulate and control these models to achieve the desired outcomes and realism, as well as to make them interactive.

Results: The radiotherapy simulator is being developed and it will involve multiple phases in order to achieve its full potential. Currently a generic treatment unit simulation is being used in order to test the functionality.

Conclusion: The computer graphics simulator has the potential to serve as an independent training aid for a department, not only for learning how to use the equipment, but also as an aid in demonstrating to patients what their treatment will involve in terms of patient setup and machine movements. The simulator also has the potential to be expanded to include other divisions in radiation medicine.

\section{Optimal skin markings for positioning in radiotherapy: Are tattoos the only option?}

\section{R. Botha}

WITS University Donald Gordon Medical Centre, Johannesburg, South Africa

Background: Reproducibility of setup is one of the most important aspects of good radiotherapy treatment. Skin markings are made at the initial simulation to facilitate this. Permanent black tattoo skin markings are the standard of care for skin marking in radiotherapy positioning; however, this practice is being called into question. The exclusive practice of placing permanent skin markings for radiotherapy does not consider the psychological impact on the patient, religious beliefs or other social impacts. There is now greater emphasis on patient autonomy and a growing number of patients want an alternative to permanent tattoos for various reasons.

Objectives: The objective of this review was to compare the available options for skin markings used for radiotherapy setup.

Method: The advantages and disadvantages of various skin marking methods will be compared, including temporary markers, such as temporary marker pens, paint markers and temporary henna tattoo skin markings, surface-guided radiotherapy, UV ink tattoos and permanent black India ink tattoos. A comparison of the durability of marking, repetition of marking session, skin reactions to markings, patient comfort and radiographer satisfaction will be done.

Results: Temporary markings can be used; however, they are not as durable as permanent tattoos and remarking periodically can lead to setup variances. When using henna, there is a time impact when remarking and therefore it is not ideal for departments with high workloads. Surface-guided radiotherapy provides an alternative to permanent tattoos; however, this is still a relatively new technology and not readily available. The cost of these upgrades in units is often very high and will likely limit the availability in the future. UV ink is a good alternative to India ink as it has the advantage of permanent marking, but the markings are not as obtrusive.

Conclusion: With current resources in most departments, permanent tattoos remain the recommended standard of care; however, UV ink may be considered instead of India ink. For patients who are strongly opposed to tattoos, temporary markings such as henna markings, paint markers and pen markers may be considered provided that the patient is adequately counselled regarding the advantages and disadvantages of different skin markings.

\section{Image-guided radiotherapy at Life Hilton Private Hospital: Radiotherapy Unit experience}

\section{N. Clarke, M. Bux, R. Coyne, N. Govender and J. Haynes}

Life Hilton Private Hospital, KwaZulu-Natal, South Africa

Background: Image-guided radiotherapy (IGRT) is the use of imaging before and/or during radiation therapy. With the development and implementation of advanced techniques such as volumetric modulated arc therapy (VMAT), intensity modulated radiation therapy (IMRT) and stereotactic radiosurgery (SRS), IGRT has become an essential prerequisite for delivering high-precision radiotherapy. It is essential however that imaging protocols are carefully developed and implemented and all staff are trained adequately. 
Method: A literature review covering the importance of pretreatment patient preparation, immobilisation and setup, as well as the different imaging modalities available and their utilisation will be covered. The roles and responsibilities of different team members will be discussed. A more practical approach will be explored as to what we currently within our own unit (at Life Hilton Radiotherapy Unit) have adopted, and helpful tips, protocols and troubleshooting ideas that we have used in our workplace will be shared.

Results: Daily imaging, if implemented correctly, ensures more accurate delivery of treatment. It also allows for the reduction of PTV margins. When limiting these margins it is important that correct image matching is performed to limit the possibility of target miss.

Conclusion: Implementing daily IGRT correctly is necessary to ensure accurate delivery of radiation treatment, especially when modulated techniques are implemented, dose escalation is performed and PTV margins are reduced. Well-developed imaging protocols need to be in place as well as sufficient training for staff needs to be conducted to ensure that treatment is delivered as intended.

\section{The evaluation of an algorithmic model, created for the image-guided radiotherapy treatment couch for integration into the Pinnacle Treatment Planning System}

\section{J.J. Botha}

\section{Groote Schuur Hospital, Cape Town, South Africa}

Background: This study was conducted as part of the MSc degree in radiography. During treatment planning, the Linac couch must be added as a structure on the plan so that its density is taken into account for dose calculation. Scripting is a programming language that supports the writing of programs for a software that automates the execution of tasks, which could alternatively be executed one-by-one by a human operator. Scripting the couch top on the Pinnacle Treatment Planning System (TPS) replaces manual contouring of the couch as a structure, thus streamlining the planning process.

Objectives: As the Varian IGRT couch is not regular in shape, the aim was to model the full couch accurately in terms of shape, dimensions and attenuation characteristics.

Methods: The actual transmission of the $6 \mathrm{MV}$ and $18 \mathrm{MV}$ Linac beams through the Varian IGRT couch top was measured at different gantry angles for three different field sizes on different parts of the treatment couch. These attenuation properties were then used to create a model of the treatment couch which can then be inserted into the treatment plans by the script. Because the shape of the couch is not regular from superior to inferior, as well as anterior to posterior, the modelling of this shape proved quite challenging and a slice-by-slice approach was used. The user specifies on the TPS where the top of the couch must be and then clicks the button to run the script. The script then determines on which part of the couch each slice is and draws the relevant couch contour, taking into account any longitudinal and lateral offsets specified by the user.

Results: The couch transmission was measured at gantry angles ranging from $120^{\circ}$ to $240^{\circ}$ through $180^{\circ}$. The couch transmission as determined by the TPS at the same gantry angles after the script was implemented was within $\pm 0.56 \%$ of the measured transmission for the $6 \mathrm{MV}$ beam and within $\pm 0.61 \%$ for the $18 \mathrm{MV}$ beam.

Conclusion: Scripting the Varian IGRT couch top on TPS was achievable and the script runs fast and reliably. The script is currently being tested clinically and will then be made available to departments running the TPS.

\section{High dose-rate cases: Thinking out of the box!}

\section{A. Heetun}

\section{Equra Health West Rand Oncology Centre, Roodepoort, South Africa}

Objective: Presentation reviewing interesting high dose-rate (HDR) brachytherapy cases performed at the West Rand Oncology Centre and Arcadia Oncology Center for gynaecological cancer.

Method: Data were collected retrospectively on patients who underwent HDR brachytherapy at the West Rand Oncology Centre. Information was gathered from electronic medical recording system (EMR), the radiation oncologist and the treating radiotherapist. Data will be presented on patients who received a modified Sorbo (Cylinder) brachytherapy treatment post-EBRT.

Results: Cases are presented with pictures and displayed in a PowerPoint presentation.

Conclusion: To present interesting out of the ordinary HDR cases that took place at this facility. This will include the preparation of the treatment, the planning of the treatment and the delivery of the treatment.

\section{Netcare pre-treatment patient-specific quality assurance for intensity modulated radiation therapy and volumetric arc therapy}

\section{H. Fourie and D.Venter} Netcare Medical Physics CoE, Netcare Ltd., Johannesburg,
South Africa

Background: The South African Standards for Quality Assurance in Radiotherapy require verification measurements for each patient undergoing intensity modulated radiation therapy (IMRT) or volumetric modulated arc therapy (VMAT). These measurements are to confirm the relative and absolute dose distributions as calculated by the TPS before the patient starts treatment. At least $95 \%$ of the evaluated points must pass the dose difference (DD) criteria by $<3 \%-4 \%$ and the distance-to-agreement (DTA) criteria by $<2 \mathrm{~mm}-3 \mathrm{~mm}$. The ideal measurement equipment and procedures have to be established to produce all the required information but still be time and cost-effective. 
Method: The Sun Nuclear DoseCHECK ${ }^{\mathrm{TM}}$, an independent Monitor Unit check software, was introduced as a surrogate for the absolute dose reproduction check. This software uses the patient CT information and 'Golden Beam' linac data to compare its own calculated dose distribution with that of the TPS. The Varian Portal Dosimetry (PD) was used for the relative dose distribution reproduction check. The IMRT/ VMAT plan is delivered on the linac and measured using the EPID. The software compares the measured fluence to the predicted fluence and also produces a composite of the individual fields. The PTW Octavius 4D phantom and 1500 array were used for absolute dose distribution measurements. All of the above equipment are capable of producing a DD and DTA analysis.

Results: The DoseCHECK ${ }^{\mathrm{TM}}$ software is based on a centralised server; hence, multiple radiotherapy sites can use the software. The dose comparisons rarely fail and if required a machine-specific model may be developed to more accurately represent the configuration of the linac. The Varian PD is not an absolute dose measurement; therefore, an MU check program or an additional absolute dose measurement is required. The PD measurement however is very quick and no additional equipment is required. The PTW Octavius 4D can produce an absolute 3D dose distribution measurement and also can perform a DD and DTA analysis in 3D. It is however time-consuming to perform a complete set of measurements (30-60 $\mathrm{min}$ ) and requires large and heavy equipment.

Conclusion: We have concluded that the DoseCHECK ${ }^{\mathrm{TM}}$ and PD are the optimal combination of solutions regarding QA, time and cost-effectiveness. The PTW Octavius 4D remains the 'gold standard' as it can produce absolute dose distributions in 3D and can be used on any linac.

\section{Empowering CHOC psychosocial support staff with knowledge and skills to support children and teenagers before and during medical treatment procedures to prevent trauma}

\section{J.A. Bence}

\section{CHOC Childhood Cancer Foundation, Cape Town, South Africa}

Background: The CHOC psychosocial support staff based in Paediatric Haematology and Oncology units across South Africa witness, on a daily basis, the debilitating, traumatic impact of invasive medical treatment procedures on the children and teenagers undergoing treatment for childhood cancer or life-threatening blood disorders. These treatments can result in a vast array of feelings, such as fear, anxiety, anger and despair, and this is often expressed as obstructive behaviour and resistance to cooperate with treatment procedures.

Method: Realising this, the CHOC psychosocial support team utilised the services of a qualified Child Life Specialist to empower them with the necessary knowledge and skills to address this problem. Each team member was provided with a 'Preparation Toolkit' to use in their work with the children and teenagers before, during and after treatment procedures.
The training methods used, both theoretical and practical, included the following: distraction, sharing of information, play and other therapeutic techniques. The importance of familiarising the children and teenagers with the medical equipment and treatment facilities was also explained.

Results: The psychosocial team observed that the children and teenagers experienced a greater sense of control over their situation, which, in turn, released stress and anxiety and assisted them to cope better. Preparing the children before procedures has proven to contribute to better physical recovery and an informal observation has also shown that as 'preparation' has become part of psychosocial service delivery, there has been a decline in families abandoning treatment.

Conclusion: Introducing 'preparation' as a part of psychosocial service delivery in $\mathrm{CHOC}$ will provide formal research opportunities.

\section{Building a psychosocial and spiritual care service for children with cancer and their families}

M. Hendricks ${ }^{1}$, C. Steenveld ${ }^{2}$, V. Thompson ${ }^{3}$, A. Andrade ${ }^{1}$, G. Kahl ${ }^{1}$, P. Farlam ${ }^{3}$, J. Kambugu-Balagadde ${ }^{1}$, S. Hendricks ${ }^{4}$, A. Rackstraw ${ }^{5}$, L. Pedersen ${ }^{6}$, D. Burger ${ }^{7}$, M. Meiring 7 , A. Davidson ${ }^{1}$ and A. van Eyssen ${ }^{1}$

${ }^{1}$ Haematology Oncology Service, Red Cross War Memorial Children's Hospital, University of Cape Town, South Africa ${ }^{2}$ Clinical Psychologist, Private Practice, Amsterdam, Netherlands ${ }^{3}$ Clinical Psychologist, Private Practice, Cape Town, South Africa ${ }^{4}$ Social Work Service, Cape Town, South Africa

${ }^{5}$ Art Therapist, Private Practice, Cape Town

${ }^{6}$ Pastoral Care Service, Red Cross War Memorial Children's Hospital, Cape Town, South Africa

${ }^{7}$ Paediatric Palliative Care Service, PaedsPal", Rondebosch Medical Centre, Cape Town, South Africa

Background: Comprehensive, coordinated psychosocial, supportive and spiritual care is an essential component of the holistic care of childhood cancer sufferers and their families.

Objectives: The authors detail the development and value of a multidisciplinary psychosocial care team as an essential adjunct to care of childhood cancer sufferers.

Methods: A historic preamble details a period during which psychosocial and supportive care was the sole province of the paediatric oncologists and social workers and describes that the process of creating a multidisciplinary psychosocial and spiritual care team has enhanced medical care.

Results: Each member of the psychosocial group describes his or her care philosophy and role in the clinical setting. We also describe the critical role of the meeting as a teaching vehicle for oncology fellows.

Conclusion: This reproducible partnership between public and private sector practitioners, designed in a resourceconstrained setting, affords the opportunity to a diverse and highly skilled group of professionals to meet the medical, psychological, social and spiritual needs of patients and families as they transition through the care journey. 


\section{Developing an in-facility intervention to manage compassion fatigue among oncology nurses, Durban, South Africa}

\section{D.L. Wentzel ${ }^{1}$, P. Brysiewicz ${ }^{1}$ and A. Collins ${ }^{2}$}

${ }^{1}$ Department of Nursing, University of KwaZulu-Natal, Durban, South Africa

${ }^{2}$ Durban University of Technology, Durban, South Africa

Background: Oncology nurses are at high risk of developing compassion fatigue and it is therefore essential to develop strategies that may assist them.

Objectives: To develop an in-facility intervention to manage compassion fatigue among oncology nurses in Durban, South Africa.

Method: The Self-Care Intervention for Oncology Nurses was developed using action research with a mixed-methods sequential explanatory design. The study was conducted with oncology nurses at three settings (a state hospital, a private hospital and a non-governmental organisation [NGO] hospice). The study comprised an integrative review, a quantitative component using a questionnaire that measured compassion fatigue, burnout and compassion satisfaction (ProQOL v5) and in-depth interviews. The oncology nurses played an active part in developing the intervention, which was then verified by experts.

Results: The intervention was developed from the findings and included psychoeducation, remembrance and support structures. An intervention booklet was designed providing psychoeducation by highlighting compassion fatigue and normalisation thereof. A remembrance tree and follow-up family phone call assisted symbolic remembrance of patients. The support group enabled oncology nurses to discuss their fears and concerns and reflect on their experiences and the effect these may have had on them.

Conclusion: Preliminary findings immediately after implementation of the Self-Care Intervention for Oncology Nurses indicated that the intervention prompted oncology nurses to become more aware of the risks of compassion fatigue, and to engage in improved self-care practices.

\section{The prevalence and genotypes of human papilloma virus in ocular surface squamous neoplasia at Dr George Mukhari Academic Hospital, Gauteng, South Africa}

\section{L.N. Mhlongo and C.M. Nkosi}

Sefako Makgatho Health Sciences University, Ga-Rankuwa, South Africa

Objectives: The purpose of this study was to determine the prevalence of human papilloma virus (HPV) in different ocular surface squamous neoplasia (OSSN) lesions, to detect the HPV genotypes in OSSN lesions and to correlate HPV and P16 ${ }^{\text {INK4a }}$ expression in OSSN lesions at Dr George Mukhari (DGM) Academic Hospital.
Method: The study was a retrospective cross-sectional observational study using archival OSSN tissue samples. A total of 58 OSSN formalin-fixed paraffin-embedded (FFPE) tissue samples classified as CIN I (5), CIN II (8), CIN III (33) and CIS (11) were randomly selected for the study by two panels of pathologists blinded. The prevalence of HPV was determined using HPV immunohistochemistry (IHC), chromogenic-in-situ-hybridisation (CISH) and polymerase chain reaction (PCR) techniques targeting the $\mathrm{L} 1$ region using the MY09/MY11 and GP5+/GP6+ primers with human $\beta$-globin (PCO3(F) and GH20(R) primers used as an internal control. The P16 ${ }^{\mathrm{INK} 4 \mathrm{a}}$ expression was detected using IHC.

Results: Human papilloma virus was detected in $18 \%(12 / 58)$ of samples using IHC, CISH for viral antigen was negative in all samples $(0 / 58), 19 \%(11 / 58)$ of samples were positive using PCR at DGM and 15.5\% (9/58) were positive using PCR at Belgium. Multiple HPV genotypes were detected in $8.6 \%(5 / 58)$ of cases. The HPV genotypes detected include HPV 16 (8), HPV 11 and 16 (5), HPV 11 (2), HPV 6 (2), HPV 81 (1), HPV 55 (1), HPV 31 (1) and HPV 67 (1). P16 1 INK4a was positive in $22.4 \%(13 / 58)$ of the OSSN cases; of these positive cases, $61.5 \%(8 / 13)$ were HPV-positive on PCR and IHC; of the HPV positive cases, the genotypes detected were HPV 6 (2), HPV 16 (4) and HPV 11 (2).

Conclusion: Human papilloma virus was positive in less than $20 \%$ of the OSSN cases, which suggests that HPV is not the cause but a co-factor in the cause of OSSN. P16 $6^{\mathrm{INK} 4 \mathrm{a}}$ was positive in more than $50 \%$ of the HPV-positive OSSN cases, which might imply that $\mathrm{P} 16^{\mathrm{INK} 4 \mathrm{a}}$ can be used as a surrogate marker for HPV, especially in the high-risk HPV types. Additional confirmatory test is essential to eliminate the risk of missing some HPV-positive tumours as HPVnegative.

\section{What to do when a competent patient refuses treatment?}

\section{A. Brummer}

Mary Potter Oncology Centre, Pretoria, South Africa

Background: As nurses we are taught to take every step possible to save a life. We are also trained to respect the patients' wishes. Sometimes these two conflict. How do we proceed?

Method: A 20-25 min slide-based presentation discussing the ethical and legal boundaries of this topic was followed. We work around evidence-based medicine that incorporates guidelines, protocols and audit.

Result: Competent adult patients are entitled to accept or reject a treatment option. The reasons do not have to be sound or rational and in fact the patients do not have to give any reason at all. Patients may even favour a treatment that is untested or even clearly detrimental to the patients' health. When a competent adult patient refuses treatment recommended by guidelines, we are bound to respect it. If we do not, we could face disciplinary action by a governing council and even possible civil or criminal proceedings. 
Informed refusal, just like informed consent, comprises three elements:

- The patient must be competent.

- He or she must have sufficient information to make a decision.

- The decision must be made voluntarily.

This involves:

- Communication: The patient has to be well informed of choices, challenges, benefits and costs. He or she needs to understand the impact of accepting a treatment versus the impact of declining the treatment, as well as the consequences.

- Patient preferences and goals need to be discussed and their healthcare professional needs to understand their thought processes.

- An acceptable solution needs to be understood by both parties and the decision needs to be documented.

- The patient may at any time change his or her mind and withdraw consent or refusal, but future healthcare decisions might be impacted by a change in their healthcare state.

Conclusion: Understanding ethical and legal parameters that healthcare professionals might face on a daily basis in an oncology setting. A few case scenarios will be used as examples to support the talk.

\section{A cohort study of treatment outcomes after radiotherapy in vulvar carcinoma patients}

\section{T. Goba-Mjwara ${ }^{1}$ and H. Simonds ${ }^{2}$}

${ }^{1}$ Division of Radiology, Stellenbosch University, Stellenbosch, South Africa

${ }^{2}$ Division of Radiation Oncology, Stellenbosch University, Stellenbosch, South Africa

Background: Carcinoma of the vulva is a rare tumour, representing about $4 \%$ of gynaecological malignancies. For locally advanced vulvar cancer, surgery often involves exenteration with colostomy or urinary diversion, causing significant physical and psychological morbidity. Neoadjuvant or primary chemoradiation is an acceptable treatment option for these patients to reduce the tumour size and minimise the extent of surgery.

Objectives: The purpose of this study was to determine the socio-demographic features and treatment outcomes in patients with vulvar carcinoma in a single institution.

Methods: A quantitative retrospective cohort study was conducted on all women diagnosed with vulvar cancer referred for radiotherapy from 01 January 2015 to 31 December 2017. Demographic and clinical data, treatment time and radiotherapy fractionation were noted. The primary end point was local control (LC) and overall survival (OS), defined as date of start of radiotherapy (RT) to date of relapse, secondary primary or death related to cancer (whichever came first).

Results: A total of 33 women were eligible for inclusion in the study. There were 22 patients who received curative treatment: definitive CRT/RT $(\mathrm{N}=17)$ and adjuvant $\mathrm{RT}$ $(\mathrm{N}=5)$. Of the 22 patients, 11 were in remission, 3 with documented local recurrence and 8 died because of unknown cause. Twelve patients received palliative treatment. The overall survival at 12 months showed that dual modality treatment resulted in a $100 \%$ survival outcome for the patients who received primary surgery followed by adjuvant radiotherapy. There was, however, no significant difference between the survival for the definitive CRT/RT (57\%) and high-dose palliative RT (52\%). The dose of RT was significant; women who received a total dose greater than $60 \mathrm{~Gy}$ had better local control compared to those who received a total dose of less than $45 \mathrm{~Gy}$.

Conclusion: Dual modality treatment had superior overall survival outcome compared to definitive chemoradiation or radiotherapy alone in this small cohort.

\section{A retrospective review of patients with stage 3 cervical cancer treated with radical radiotherapy at Universitas Hospital Annex from 2001 to 2010}

\section{Rothman and H. Napo \\ University of the Free State, Bloemfontein, South Africa}

Objective: To determine the disease-free survival and the overall survival in patients with stage 3 cervical cancer treated with concurrent chemoradiation.

Method: A retrospective analytical cohort study was conducted. We reviewed the files of 393 patients with stage 3 cervix cancer who were treated at the Universitas Hospital Annex between 2001 and 2010. Data collected included the total dose of external beam radiation and brachytherapy, the total number of concurrent chemotherapy cycles, performance status, HIV status and CD4 counts.

Results: The 5-year survival was $86.4 \%$ and the 5-year disease-free survival was $66.3 \%$. The total radiation dose (EQD2) did not make a significant difference in the overall survival ( $p=0.48 ; \mathrm{HR} 0.98$ ) or the disease-free survival ( $p=0.19$; HR 0.98). Concurrent chemotherapy did not affect the overall survival ( $p=0.99$ ) but had a significant impact on the disease-free survival $(p=0.03)$. The number of chemocycles also impacted the disease-free survival ( $p=0.03$; HR 0.73 ). The age of the patients was significantly associated with disease-free survival $(p<0.01 ;$ HR 0.97$)$ but not with the overall survival ( $p=0.84$; HR 1.00).

Conclusion: Whether the patients received chemotherapy and the number of chemotherapy cycles had a statistically significant impact on the disease-free survival but not on the overall survival. The total dose of radiotherapy (EQD2) did not have a significant impact on either disease-free or overall survival. 


\section{Sexual quality of life in locally advanced cervical cancer patients treated with definitive concurrent chemoradiation}

\section{P. Pillay ${ }^{1}$, C. Minnaar ${ }^{1}$ and Kotzen J² \\ ${ }^{1}$ University of the Witwatersrand, Johannesburg, South Africa \\ ${ }^{2}$ Wits Donald Gordon Medical Centre, Johannesburg, \\ South Africa}

Background: Research on radiation toxicity has primarily focused on symptoms related to organs such as the bladder, bowel and rectum; however, the vagina is both the target and an organ at risk.

Objectives: The purpose of this study was to describe the sexual quality of life $(\mathrm{QoL})$ of patients treated with chemoradiation for locally advanced cervical cancer.

Method: This is a retrospective analysis of prospectively collected data from a phase III randomised controlled trial investigating the benefits of the addition of hyperthermia to chemoradiation from January 2014 to June 2017. A total of 76 participants from the control arm with a median follow-up of 12 months were included in the analysis. QoL was prospectively assessed using EORTC QLQ-CX24 Quality of Life questionnaires at baseline, then every 3 months posttreatment during the first year, and every 6 months in the second year.

Results: Sexual worries (i.e. sex would be painful), and poor body image, were present in $60 \%$ of participants at baseline and remained constant for the first year posttreatment but decreased to $35 \%-40 \%$ in those who survived beyond a year. Of the participants, $11 \%(n=8)$ reported being sexually active at baseline, but this doubled by 6 months post-treatment $(n=17)$ and increased to $30 \%$ after 9 months post-treatment. Twenty-one per cent of participants under 30 years, 22\% aged $31-50$ years and $10 \%$ over 50 years of age reported having sex in the last 4 weeks. Out of the 72 reports of sexual activity, pain during intercourse was reported by $65 \%$ of participants, while $77 \%$ found intercourse enjoyable. Of the participants, $51 \%$ found it both enjoyable and painful, $26 \%$ enjoyed sex without pain and $14 \%$ who did not enjoy intercourse reported pain. The main vaginal symptom was discharge that was almost two times more prevalent than vaginal discomfort throughout the first two years post-treatment $(71 \%$ vs. $43 \%$ at baseline; $40 \%$ vs. $27 \%$ at 6 months; $23 \%$ vs. $14 \%$ at 18 months). However, there was a clear and noteworthy improvement over time.

Conclusion: The sexual QoL of participants was greatly impaired before treatment and their concerns persisted throughout the first year, with an improvement in the subsequent year only. Providing care on a daily basis without an in-depth understanding of what is most meaningful to patients only widens the gap in care. These findings can help health professionals to shape expectations by paying attention to concerns, and to reassure patients that there are improvements over time.

\section{A retrospective study of treatment outcomes of cervical cancer in young women treated at a single institution}

\section{Njovu and H. Simonds \\ Department of Radiation Oncology, Stellenbosch University,} Stellenbosch, South Africa

Background: Cervical cancer affects mostly young women from poor countries and disadvantaged populations. There is limited information available to specifically outline the presenting features and treatment outcomes in young patients treated for cervical cancer.

Objectives: The aim of this study was to evaluate the demographics and treatment outcome in young women under the age of 40 years with cervical cancer treated at a single institution in South Africa.

Methods: A retrospective study that reviewed medical records of patients younger than 40 years with cervical cancer referred for radiation from January 2015 to December 2018 was followed. The MOSAIQ ${ }^{\circledR}$ patient management system was used as the source of patient names. Data collected included patient demographics, HIV status, stage, treatment and survival outcome.

Results: During the study period, 96 patients under the age of 40 years were referred for radiotherapy. The median age was 33 years $(24-38$ years) and $15.6 \%(n=15)$ were under the age of 30 . Overall, $36.5 \%$ were HIV-positive. Disease characteristics included $91.7 \%$ squamous cell carcinoma on histology and $65.6 \%(n=63)$ were stage IIIB and above. The very young patients (under 30 years) had a higher proportion of presenting with advanced stage disease $(73.3 \% ; n=11)$. Seventy-one patients $(74 \%)$ received radical CCRT, RT or adjuvant CRT and 77 patients $(80.2 \%)$ received $>40 \mathrm{~Gy}$. HIV-negative patients were significantly more likely to be prescribed curative therapy $(82 \%$ vs. $60 \% ; p=0.018)$. Of the 71 patients who received radical therapy, $17(23.9 \%)$ had a recorded date of death by the end of study, with no significant difference by HIV-status.

Conclusion: The young women presenting with cervical carcinoma at our institution had a high prevalence of HIV and advanced stage. Although HIV status impacted treatment intent, more than $80 \%$ of the cohort received a high dose of radiation.

\section{Gestational trophoblastic neoplasm: A retrospective review in a South African population}

\section{K. Pemberton and N. Fakie}

University of Cape Town, Cape Town, South Africa

Background: Gestational trophoblastic diseases comprise partial and complete hydatidiform mole, persistent or invasive mole, choriocarcinoma and placental trophoblastic tumours. Partial and complete hydatidiform moles are considered premalignant forms of the disease, whereas 
persistent or invasive mole, choriocarcinoma and placental site trophoblastic tumours are malignant and therefore termed gestational trophoblastic neoplasms. Gestational trophoblastic diseases have an estimated incidence of 1-3:100 pregnancies for complete hydatidiform mole, 3:1000 pregnancies for partial hydatidiform mole, and 1:50 000 deliveries for choriocarcinoma and placental site trophoblastic tumours in the United Kingdom. However, it is most common in Asian populations. The epidemiological factors, treatment strategies and outcomes are not known in the South African population.

Objectives: To identify the epidemiological factors, presenting symptoms, management strategies and outcomes of patients treated for gestational trophoblastic neoplasms between 2010 and 2017 at the Groote Schuur Hospital, Cape Town, South Africa.

Method: A retrospective review will be conducted on a study population of 50 female patients who presented with and were treated for gestational trophoblastic disease, based on serum beta human beta chorionic gonadotropin levels and histological diagnosis at the Groote Schuur Hospital from 01 January 2010 to 01 January 2017. Data will be obtained from the Department of Radiation Oncology, Groote Schuur Hospital Cancer Registry.

Results: Results will include the identification of incidence rates, significant epidemiological factors, presenting symptoms and outcomes identified by the number of consolidation cycles and remission rates. Variables will be assessed using correlation analysis and data will be compared with previous data using parametric testing.

Conclusion: At the end of the study, a better understanding of the epidemiology, management and outcomes of gestational trophoblastic disease in a South African population will be attained. This will be influential as it would outline the differences from and similarities to international presentations, protocols of management and prognoses.

\section{Training radiation oncologists for low- and middle-income countries: Have we made any impact? A 20-year study at the University of Cape Town}

\section{J. Parkes ${ }^{1,2}$ and Z. Mohamed ${ }^{1,2}$}

${ }^{1}$ University of Cape Town, Cape Town, South Africa

${ }^{2}$ Groote Schuur Hospital, Cape Town, South Africa

Background: In 1999, the Radiation Oncology Department at the University of Cape Town (UCT) embarked on a policy of implementing training for international radiation oncologists.

Objectives: The purpose of this study was to evaluate the success of the programme implemented with respect to the origin of the professionals trained, how successful they were at completing the programme and their current positions.

Method: All international doctors and supernumerary registrars registered in the Department of Radiation Oncology at UCT from 1999 to 2019 were included in the study.
Evaluation was done according to countries of origin, short period or full training, whether the full training registrars stayed for the intended duration of study, whether they were able to pass the Part 2 South African examinations in Radiation Oncology by the end of their training time and whether they returned to their country of origin after completion of training and are still working there.

Results: A total of 39 supernumerary doctors were registered between 1999 and 2019. Thirty-one of these were international doctors and eight were registrars from other regions in South Africa. Of the 31 international registrars, 28 were registered for full training and 3 for short visits of 6 months. Of the 28 registered for full training, 10 are currently still in the programme, 16 have completed the programme and $2 \mathrm{did}$ not complete the programme. The trainees were from 13 different countries. Only one registrar did not pass the Part 2 CMSA examinations by the end of training. Of the 16 who completed the programme, seven are currently heads of department in their home countries, six are working in their own countries, one has emigrated, one is working in another country in Africa and one is not currently working but plans to return to his country of origin and work. Of the eight local registrars who attended the department as part of their training, five passed Part 2 CMSA examinations at completion of training and three have never passed. Of the five who are specialists, four specialists work in their province of origin, but two are in private practice. One relocated to another province.

Conclusion: The international training programme at UCT has successfully trained radiation oncologists from many countries in Africa and beyond. Few international registrars do not successfully complete the programme. Most international registrars are still in practice in their countries of origin and a high number of them are heads of department. Of the local registrars who completed part of their training in the department, the pass rates are slightly lower, but many stay in the state sector working in oncology. Overall, the programme shows a high impact.

\section{The role of surgery and radiotherapy in the management of neuroblastoma in South Africa}

J. Van Heerden ${ }^{1,2}$, G.P. Hadley ${ }^{3}$, M. Hendricks ${ }^{4}$, J. Geel ${ }^{5}$, B. Sartorius ${ }^{3,6}$, J. Du Plessis ${ }^{7}$, A. Büchner ${ }^{8}$, G. Naidu', B. van Emmenes $^{9}$ A. van $\mathrm{Zyl}^{1}$ and M. Kruger ${ }^{1}$

\footnotetext{
${ }^{1}$ Stellenbosch University, Stellenbosch, South Africa ${ }^{2}$ Antwerp University Hospital, Antwerpen, Belgium ${ }^{3}$ University of KwaZulu-Natal, Durban, South Africa ${ }^{4}$ University of Cape Town, Cape Town, South Africa ${ }^{5}$ University of the Witwatersrand, Johannesburg, South Africa ${ }^{6}$ University of Washington, Seattle, WA, United States of America ${ }^{7}$ University of the Free State, Bloemfontein, South Africa ${ }^{8}$ University of Pretoria, Pretoria, South Africa ${ }^{9}$ Frere Hospital, East London, South Africa
}

Background: Local therapies are essential in the management of neuroblastoma (NB) regardless of risk classification. A multi-institutional retrospective study across South Africa 
was conducted to evaluate the impact of surgery and radiotherapy on outcomes in NB patients.

Methods: Patients treated between January 2000 and December 2014 in nine POUs were included. Demographic data, disease stage, risk stratification, degree of surgery, the administration of radiotherapy and outcome were collected after custodian consent and individual consent waivers. Kaplan-Meier curves, Cox regression models and Fischer's exact test were used for data analysis and calculation of the 2-year overall survival (OS).

Results: Data from 390 patients were included. The median age was 39.9 months (standard deviation [s.d.] 36.8, range 0-201 months). The majority of patients $(75.6 \%)$ presented with high-risk (HR) disease. Only $42.8 \%$ received treatmentrelated surgical interventions across all risk groups. Of patients with very low-risk (VLR) disease, 88.2\% were operated, $76.9 \%$ of low-risk disease (LR), $70 \%$ of intermediate-risk (IR) and $35.6 \%$ of HR. Radiotherapy was administrated to $16.7 \%$ of IR and $16.3 \%$ of HR patients. HR patients achieved a $25.4 \%$ postinduction complete metastatic remission (MR), while $82.6 \%$ $(n=62)$ were operated. Incomplete MR was achieved in $71.5 \%$ of patients, but only $18.5 \%(n=39)$ were operated. In HR disease $(n=295)$, completely resected (CR) tumours had a 2-year OS of $46.4 \%$, incomplete resected (ICR) tumours $51.4 \%$ and unoperated tumours (UT) $16.2 \%$ ( $p<0.001,95 \%$ CI). In IR disease $(n=30)$, CR had a 2 -year OS of $83.3 \%$, ICR $70.1 \%$ and UT $42.9 \%(p<0.001,95 \%$ CI). In LR disease $(n=26)$, CR had a 2-year OS of $84.4 \%$, ICR $100 \%$ and UT $42.8 \%$ ( $p<0.001,95 \%$ CI). In LR disease, CR and ICR had a 2 -year OS of $100 \%$, but UT $50 \%(p<0.001,95 \% \mathrm{CI})$. Only two patients $(1.2 \%)$ died from surgical complications. In HR disease, patients who received tumour bed radiotherapy had a 2-year OS of $42.3 \%$ versus $24.1 \%$ of those with no radiotherapy. In IR disease, who received tumour bed radiotherapy had a 2-year OS of 75\% compared to no radiotherapy of $63.4 \%$ ( $p<0.001,95 \% \mathrm{CI})$. Even in HR disease, patients who received radiotherapy without surgical interventions had an improved 2-year OS of $25.8 \%$ as opposed to $17.3 \%$ in patients who did not receive local therapies $(p<0.001, \mathrm{CI} 95 \%)$. On multivariate analysis, stage $(p<0.0001,65 \% \mathrm{CI})$, risk classification $(p<0.0001,95 \%$ $\mathrm{CI})$, post-induction complete remission rate $(p<0.001,95 \% \mathrm{CI})$, degree of surgical resection $(p<0.01,95 \% \mathrm{CI})$ and radiotherapy administration $(p<0.001,95 \% \mathrm{CI})$ were identified as significant prognostic factors.

Conclusion: Both complete and incomplete surgeries provide a survival advantage to neuroblastoma patients, who achieved $\mathrm{mCR}$. Monotherapy radiotherapy benefited local control. Both treatment modalities should be implemented in settings without access to autologous transplant or targeted therapy.

\section{Complications in the placement of central lines for paediatric oncology patients at Universitas Academic Hospital, from 1992 to March 2018}

K. Magooa, L. Mokone, P. Ndovo, R. Rampeta, K. Tsilo, E. Brits and G. Joubert

University of the Free State, Bloemfontein, South Africa
Background: Central venous access devices (CVADs) such as external tunnelled cuffed catheters (Hickman Lines) are notoriously associated with complications such as central line-associated infections and, more severely, systemic sepsis.

Objectives: To determine the complications of central lines for chemotherapy in paediatric oncology patients treated at the Paediatric Haematology and Oncology Unit at Universitas Academic Hospital in Bloemfontein from 1992 to March 2018.

Method: This was a retrospective descriptive study. The study population included paediatric oncology patients, aged 16 years and younger, who received CVADs and treated at the Paediatric Haematology and Oncology Unit at Universitas Academic Hospital in Bloemfontein. Information was collected from their database.

Results: There were 293 lines that were inserted with a median age of 64.7 months. There were no in situ local complications in 183 (62.5\%) of the lines, while 39 (13.3\%) showed sepsis, and the rest of the lines had scientifically insignificant complications. Of the 39 that had line sepsis, $23 \%$ had no systemic sepsis and $61.5 \%$ were neutropenic and septic. One hundred ninety patients had systemic symptoms of sepsis and accompanying neutropenia, of whom 128 (67.4\%) did not develop in situ line complications. Eighty-five $(44.7 \%)$ lines were removed because it was the end of treatment and only $31(16.3 \%)$ lines were removed as a result of line sepsis.

Conclusion: Despite the study population having characteristics that make them more prone to infections, there was a significantly low incidence of line sepsis complications $(13.3 \%)$. The special care taken intraoperatively and the meticulous aseptic handling and maintenance post-operatively can be the reason for the low infective complication values.

\section{Poor prognostic features of children diagnosed with osteosarcoma over a 20 -year period in a hospital in Johannesburg}

\section{T. Ngcana}

\section{Chris Hani Baragwanath Hospital, Johannesburg, South Africa}

Background: Survival rates of South African children with osteosarcoma (OS) are poor, but prognostic factors have not been elucidated in an African setting.

Objectives: To determine the overall survival rate and prognostic factors in children with OS at Charlotte Maxeke Johannesburg Academic Hospital (CMJAH).

Methods: A retrospective review was conducted on children with OS from 1990 to 2010. Descriptive statistics, KaplanMeier survival analysis and Cox logistic regression were performed.

Results: Fifty-five files were available for analysis. The median symptom duration was 90 days and the median age was 12.1 years. The majority of patients had poor prognostic features: 47 (85\%) had masses larger than $8 \mathrm{~cm}$ in diameter, 
all patients had high alkaline phosphatase and 46 (83.6\%) had elevated lactate dehydrogenase (LDH). Histology included osteoblastic (37/55) and chondroblastic OS (9/55). Forty-three (78.2\%) patients had low body mass index (BMI) at presentation. Forty-one patients were treated with ifosfamide, doxorubicin, methotrexate and cisplatin and one patient was on a hybrid protocol. Thirteen patients (25\%) did not receive chemotherapy and four patients declined surgery. The 5-year survival rate of those treated with curative intent was $42 \%$. Causes of death included progression of disease $(22 / 42)$ and treatment complications $(5 / 42)$. On univariate analysis, site of primary tumour $(p=0.044), \mathrm{LDH}(p=0.011)$ and stage ( $p=0.0001)$ were found to have impact on survival, while multivariate analysis confirmed only stage $(p=0.0001)$ and unknown LDH level ( $p=0.011)$ as significant.

Conclusion: Advanced disease at presentation, which is a modifiable factor, was common and impacted negatively the overall survival.

\section{Paediatric pulmonary leiomyosarcoma}

\section{K. Coopasamy, R. Thejpal, Y. Goga, B. Neethling and H. van Staaden}

Department of Paediatrics and Child Health, Nelson R Mandela School of Medicine, Inkosi Albert Luthuli Central Hospital, Durban, South Africa

Background: Inkosi Albert Luthuli Central Hospital services the entire province of KwaZulu-Natal which has a large burden of paediatric HIV infections. Leiomyosarcomas are rare and we have not seen the increase in the paediatric AIDS defining leiomyosarcomas described in developed countries. With wider HAART coverage, HIV-related cancers would drop.

Method: Case presentation of primary pulmonary leiomyosarcoma is extremely rare and has poor outcome if not completely resected. An 8-year-old girl presented with a non-productive cough for 6 months and constitutional symptoms. Examination revealed severe respiratory distress with features of dense consolidation on her right chest. She was commenced on anti-TB treatment. Gene expert returned negative. CXR showed large opacification right side of chest. She developed severe upper airway obstruction, requiring intubation and supportive ventilation on transfer. Computed tomography (CT) chest revealed a large heterogeneously enhancing solid mass with areas of breakdown measuring $13 \mathrm{~cm} \times 10 \mathrm{~cm} \times 10 \mathrm{~cm}$, extending from the base of the neck into the right thoracic cavity. The mass abutted and encased vessels. A percutaneous tru-cut biopsy revealed a muscle tumour, with features of leiomyosarcoma (positive for Desmin and SMA). The cardiothoracic team was unable to consider resection in view of major vessel encasement. Radiation of the tumour was not considered to be a practical alternative. She was given chemotherapy (two cycles of adriamycin, bleomycin, vinblastine and dacarbazine). Because of poor response the patient was given one cycle of ifosphamide and etoposide. The patient failed extubation multiple times because of functional obstruction of the mass.
Repeat imaging revealed poor response to chemotherapy. A multidisciplinary team decided to palliate and stop further chemotherapy. Her mother was extensively counselled. She was then transferred to the base hospital with an advanced care plan and a ventilation weaning protocol. The patient demised at the base hospital while weaning 3 months after diagnosis.

Results: Primary pulmonary leiomyosaroma occurs in $<0.5 \%$ of all pulmonary tumours, with a reported 5-year survival of $60 \%$ if completely resected. Surgery is the definitive mode of management. If early complete resection is achieved, the 5-year survival is $50 \%$. Adjuvant radiotherapy and chemotherapy are recommended in incomplete resection or unresectable tumours. Early referral will decrease mortality in this rare malignancy.

\section{Developing a national nutritional study in paediatric oncology units in South Africa}

\section{J. Schoeman ${ }^{1}$, E.J. Ladas ${ }^{2}$, P.C. Rogers ${ }^{3}$, S. Aryal ${ }^{2}$ and M. Kruger ${ }^{1}$}

\author{
${ }^{1}$ Stellenbosch University, Stellenbosch, South Africa \\ ${ }^{2}$ Columbia University Irving Medical Centre, New York City, NY, \\ United States of America \\ ${ }^{3}$ University of British Columbia, Vancouver, Canada
}

Background: Globally more than 160000 children and adolescents are diagnosed with cancer each year. The majority $(80 \%-85 \%)$ live in low- or middle-income countries (LMICs) and up to $50 \%$ of these children are malnourished.

Objectives: To investigate the incidence and severity of malnutrition, food security and poverty in children diagnosed with cancer over a 3-year period in South Africa. The association of these variables with underlying cancer, 2-year overall survival (OS) and event-free survival (EFS) was investigated.

Method: This is a prospective cohort study in state-funded paediatric oncology units (POUs) in South Africa for a period of 3 years. All newly diagnosed children with cancer were enrolled in the study within $72 \mathrm{~h}$ after diagnosis with consent obtained. Nutritional assessment was done at diagnosis and monthly during treatment: anthropometry, biochemistry and clinical assessment. This was repeated 3-monthly after treatment till the end point. Personal interviews were conducted with parents to complete the Hunger Scale Questionnaire and Poverty Assessment tool for determining socio-economic circumstances at home.

Results: To date 52 patients were enrolled in the study. The preliminary results show $57.7 \%$ (30) were boys and $43.3 \%$ (22) were girls, with an age range of 8-177 months. One interesting fact is that $18 \%(9)$ were premature babies. The majority of patients are Africans $(67.3 \% ; 35)$, mixed race $(26.9 \% ; 14)$ and white $(5.8 \% ; 3)$. Most of the children live in Western Cape $(42.3 \% ; 22)$ and Mpumalanga (41.4\%; 21), while $15.4 \%$ were from Gauteng and $1.9 \%$ (1) were from North West province. 
Conclusion: This is the first study in sub-Saharan Africa that will systematically investigate nutritional status at diagnosis, food insecurity and risk for poverty in children with cancer and the association with OS and EFS. The findings will assist in creating a comprehensive supportive care plan for children with cancer in future.

\section{Volumetric modulated arc therapy: A clinical and patient-orientated experiential review}

\section{N.S.Fourie, L. Kruger, M. Neethling and M.Wagner}

\section{Equra Health, Panorama Oncology Unit, Cape Town, South Africa}

Background: The novel radiation technique, volumetric modulated arc therapy (VMAT), can offer improved sparing of normal tissues and target volume coverage, whilst achieving highly conformal dose distributions when compared with conventional radiotherapy techniques. Globally the clinical use of VMAT is significantly increasing, with additional advantages including reduced treatment delivery time, when compared with intensity modulated radiation therapy (IMRT).

Objectives: To provide feedback from clinical oncologists, radiation therapists and retrospective patients with regard to experience related to the VMAT treatment modality.

Method: Reviews were obtained from radiation oncologists regarding planning experience, including the draw of tumour volumes, duration of planning, plan reviews and the treatment of related side effects. Patients who received previous IMRT and were re-treated with VMAT within the past 5 years were interviewed. Feedback was obtained from personal experience including daily treatment time, the comfort of treatment duration and devices, as well as management of side effects during and after treatment. Radiation therapists were questioned to obtain opinions on the use of VMAT as a treatment modality with regard to scheduled daily time slots, imaging and daily radiation setup.

Results: The results of combined feedback tended to be in favour of the VMAT modality.

Conclusion: The VMAT modality remains a relatively novel technique, with published data focused on dosimetric planning studies with limited clinical outcomes. Although the VMAT modality holds various distinct advantages, it cannot be considered as a universal solution in all clinical cases.

\section{A retrospective report on the use of cone beam computed tomography for adaptive radiotherapy for lung cancer}

\section{J. Thompson and M. Bassa}

Equra Health, Cape Town, South Africa

Background: Kilovoltage cone beam computed tomography $(\mathrm{CBCT})$ in room imaging is used to provide superior soft tissue information. For lung imaging, it has become a fast growing technique as we move from standard to complex delivery techniques, treatment verification and inter-fraction adaption; it has become essential especially when treating lung cancer.

Objectives: The objectives of this study were to provide us with the necessary information needed to recognise and implement changes in departments equipped with this imaging modality. Adequacy of the PTV margin is important particularly in lung cancer cases because of radiation-induced tumour changes as well as geometric uncertainties considering the low stability of the treatment area.

Method: A quantitative retrospective study was conducted on patients presenting at a radiotherapy department. A convenience sample size of 15 patients over the past 2 years was selected. Patients 18 years or older with previously untreated small lung cell cancer or non-small cell adenocarcinoma of the lung were considered. A total prescribed dose of $60 \mathrm{~Gy}$ in 30 fractions was followed. Three aspects involving these cases were looked at: (1) clinical cases involving volume expansion and how the treatment plan was adapted; (2) cases that could have been adapted during the treatment (in the case of PTV adjustments following a replan, the difference of the PTV from the original plan to the adapted plan was measured); and (3) volumes that required no re-plan but had to be monitored for geometric uncertainties.

Results: Fifteen patients' CBCT had been looked at retrospectively. Of the 15 patients, $9(60 \%)$ had a tumour response and could have been re-planned, $3(20 \%)$ patients showed no change to the tumour throughout the treatment but had to be monitored for geometric uncertainties and $3(20 \%)$ patients had been re-planned because of their rapid tumour growth not being included in the PTV. Radiation changes and the growth of the tumour had been noticed in majority of the patients; however, adequate PTV margins had been used to ensure that the volume is still covered.

Conclusion: In radical radiotherapy (RT) for lung cancer, daily CBCT allows us to use IGRT to support adaptive RT and has the potential to reduce PTV margins, which would result in improved outcomes for lung cancer patients. Furthermore, it provides us with useful information to prevent geometric uncertainty and ensure adequate tumour coverage especially.

\section{Optimising prostate conformal radiotherapy planning with a prioritised list of parameters}

\author{
J.M.Steyn ${ }^{1}$, F.C.P du Plessis² and W.I.D Rae ${ }^{2}$ \\ ${ }^{1}$ Department of Medical Physics, Equra Health, Cape Town, \\ South Africa \\ ${ }^{2}$ Department of Medical Physics, University of the Free State, \\ Bloemfontein, South Africa
}

Background: Treatment planning is aimed at delivering sufficient radiation dose to the tumour, while sparing the surrounding, uninvolved tissues. The planning of prostate conformal radiotherapy (CRT) has been widely standardised. However, evaluation varies considerably across planning centres. 
Objectives: The aim of this study was to determine a prioritised list of physical and dosimetric (biological) parameters for evaluation, and thus improvement, of CRT treatment plan quality.

Methods: Details of one patient requiring prostate radiotherapy were sent to $19 \mathrm{XiO}$ treatment planning systems, and CRT planning was done by 19 planners. Four physical parameters (number of beams, opposing fields, field size and wedge) and eight biological parameters (heterogeneity, conformity, maximum, minimum and mean dose, $\mathrm{V}_{50}$, and $\mathrm{V}_{65}$ for the rectum and $\mathrm{V}_{5}$ for the bilateral femora) were extracted from each plan. Verification of the dose delivery was performed with Gafchromic film within a Rando phantom. Using principal component analysis (PCA), a list of evaluation parameters was obtained. Several plans were used to verify the application of this list.

Results: The PCA indicated that the biological parameters best predicting treatment plan quality are heterogeneity index, minimum dose and rectal $\mathrm{V}_{65}$. Dose verification with the film showed $>90 \%$ agreement. No alteration to the biological parameters was required. Using the proposed parameters showed 22\% improvement in the indices, $5.6 \%$ improvement in the ICRU dose limits and an $8 \%$ decrease in small bowel dose. A 3.5\% increase in the dose to the rectum, bilateral femora and bladder was observed.

Conclusion: The number of beams and field size should be used to improve the biological parameters. A verified protocol for planning evaluation was developed and implemented using the identified parameters. This study is being expanded and extended to include more plans.

\section{Efficiency index: Reporting method for stereotactic treatment plans}

\section{N.S. Fourie ${ }^{1}$ and A. Dreyer ${ }^{2}$}

${ }^{1}$ Equra Health, Panorama Oncology Unit, Cape Town, South Africa ${ }^{2}$ Cancer Care, Panorama Oncology Unit, Cape Town, South Africa

Background: Stereotactic radiotherapy is defined as an external radiation beam, which is precisely aimed at a tumour. A very high and intense dose is delivered, making a steep dose fall-off essential to minimise normal tissue toxicity. Dose sparing has been highlighted again in the ICRU 91 report, which focused on stereotactic treatments. In 2018, Dimitriadis et al. have published a novel metric: the efficiency index $\left(\eta_{50 \%}\right)$, which is used to determine the dose sparing and dose fall-off. It focusses on the relation of useful energy over input energy. Useful energy is referred to as the energy that is needed to treat the tumour. Thus, obtaining a higher efficiency index indicates more energy (dose) is deposited into the tumour and less is wasted. At Panorama Oncology Unit we have started to use the efficiency index as a reporting method on neuro-stereotactic radiotherapy patients.

Objectives: Efficiency index has been implemented in our unit as a reporting method on neuro-stereotactic patients.
Method: Currently our dose prescription for neurostereotactic patients is done at $80 \%$ at $6 \mathrm{MV}$, meaning that the prescribed dose should fall onto the $80 \%$ isodose level, covering the planning tumour volume. Internationally this prescription dose level varies to obtain a steep dose fall-off. Firstly, the efficiency index value, expressed as a percentage, was calculated retrospectively for eight of our treatment plans, comparing with the internationally published values. Secondly, the prescription dose level was changed from $80 \%$ to $50 \%$ using the same eight treatment plans. Lastly, three treatment plans were re-optimised for $10 \mathrm{MV}$.

Results: Internationally, the efficiency index value varies between $30 \%$ and $45 \%$. Our calculated efficiency index values fall between $20 \%$ and $48 \%$. It seems that the $50 \%$ dose prescription level results in a steeper dose fall-off, however, increasing the hotspot. Re-optimising with a different energy did not lead to a significant difference in the efficiency index percentage.

Conclusion: The efficiency index is a useful and easy tool to compare treatment plans. This index is sensitive to conformity and dose fall-off. It can be used and implemented with any treatment planning system. However, an index cannot replace a clinical decision and evaluation.

\section{A novel treatment planning model for total skin electron irradiation}

\section{K.N Sachse, L.J. Strauss and W. Shaw}

\section{University of the Free State, Bloemfontein, South Africa}

Background: Total skin electron irradiation (TSEI) is a standard treatment technique for mycosis fungoides. No clinical treatment planning systems exist for treatment planning at large source-to-skin distances (SSD) without the use of electron applicators. We have developed an electron beam characterisation model for an Elekta ${ }^{\circledR}$ Precise linear accelerator using the EGSnrc/DOSXYZnrc Monte Carlo (MC) code to perform treatment planning and have dosimetrically verified the model on an anthropomorphic phantom.

Method: A dual point-source DOSXYZnrc beam characterisation model was constructed for a 4-, 6- and $8 \mathrm{MeV}$ electron beam, collimated to a $40 \times 40 \mathrm{~cm}^{2}$ field at isocentre. Electron and photon beam characteristics were extracted from simulated phase space files. Fluence spectra for each energy were modelled independently using mathematical functions to dosimetrically match measured off-axis ratios (OARs) at multiple SSDs in water. In addition, abutted two-dimensional (2D) array measurements at isocentre in water were compared with simulated 2D dose distributions. Output factors (OFs), central axis (CAX) and off-axis (OAX) percentage depth dose (PDD) curves were also verified for SSDs between $100 \mathrm{~cm}$ and $200 \mathrm{~cm}$. An in-house full-body anthropomorphic phantom was made and computed tomography (CT) scanned, requiring two half-body scans to be joined together in a single wholebody CT set. A discrete beam arc with various arc increments was simulated with the phantom at $180 \mathrm{~cm} \mathrm{SSD}$, covering the entire phantom using an energy-dependent optimal separation 
distance between four planes, each with individual beam central axis levels from head to toe. Dose evaluation was performed on ProSomaCore after converting MC 3D dose data to DICOM format and compared with in vivo measurements.

Results: The beam characterisation models produced CAX and OAX PDD curves, OARs and OFs to within 2\% of measured data, whereas 2D dose distributions complied with a $2 \% / 2 \mathrm{~mm}$ dose/distance-to-agreement criterion in both water and the phantom. Improved dose uniformity at the depth of maximum dose in the anthropomorphic phantom was observed when the number of beam angles was increased. Because of the variation in phantom contours (hence SSD variations), hot-and-cold spot formation across the phantom was identified, and consequently beam weighting could be optimised to improve dose uniformity along treatment planes from head to toe.

Conclusion: The MC beam model compared to within $2 \%$ of measured data. Increasing the number of beam angles for total skin electron irradiation (TSEI) improves dose uniformity, especially in large-diameter treatment regions. Dose visualisation and analysis allowed beam weight optimisation for a beam model that can be used for patientspecific treatment planning.

\section{Treating Chaos the Lion and other cats: A roaring experience!}

\section{H. Reynolds}

Equra Health Arcadia Oncology Centre, Pretoria, South Africa

Background: This wonderful story as the title mentions made news worldwide! Chaos the Lion was referred to Arcadia Oncology Centre. This private unit often treats animals, especially pets; however, treating a lion was a first.

Objectives: To share the amazing and overwhelming experience of treating the king of the jungle! This unit's animal treating CV also includes a leopard and a cheetah. These case studies will also be shared during the presentation.

Method: Information from these case studies will be presented as an oral presentation. The animal treatment technique, prescription and challenges will be discussed.

Results: Pictures of the animals' treatment will be shared during the presentation, as well as their treatment outcome on follow-up.

Conclusion: To share this once-in-a-lifetime story of Chaos the Lion and other cats with the oncology community. All these case studies have been memorable experiences for all the staff involved.

\section{The Cape Town access to care course: What we have learnt and what the future holds}

N. Vowles ${ }^{1}$, H. Burger ${ }^{2,3}$, B. Wyrley-Birch ${ }^{1}$ and J. Parkes ${ }^{2,3}$

Acknowledgements: S. Berz ${ }^{3}$, J. Groll ${ }^{3}$ and J.M Valentim ${ }^{3}$
${ }^{1}$ Cape Peninsula University of Technology, Cape Town, South Africa ${ }^{2}$ University of Cape Town, Cape Town, South Africa

${ }^{3}$ Groote Schuur Hospital, Cape Town, South Africa

${ }^{4}$ Varian Medical Systems, Steinhausen, Switzerland

Background: Increased access to radiotherapy treatment units, treatment planning systems and appropriate dosimetry equipment coupled with a need for the implementation of three-dimensional (3D) conformal radiotherapy (3DCRT) protocols and the appropriate skills training for staff is an ongoing challenge throughout Africa. The Groote Schuur Hospital Access to Care (A2C) course focuses on moving from two-dimensional (2D) to 3DCRT. The A2C Centre is a technology-based teaching platform for the radiation oncology treatment team. It is a joint collaborative training initiative between Varian Medical Systems, UCT and CPUT.

Methods: Four teams attended each course and were asked to complete surveys before, during and after the integrated 3-week course. Consent was obtained from the participants to use the information. The surveys of the six courses spanning 2015-2019 (72 participants) will be analysed and discussed in terms of pre- and post-course knowledge and experience.

Results:

- All participants agreed that the course added value to their application of radiotherapy treatment knowledge. Certain valuable learning points identified included planning of specific sites, practical application of planning and contouring, theimportance of ethical multidisciplinary teamwork, protocols and quality assurance.

- Before attending the course, $36 \%$ of respondents felt hampered by limited dedicated time for planning and contouring in their own departments. Each course provided 35 dedicated hours of practical contouring and planning with assistance, which resulted in $16 \%$ improvement in the need for practising these skills.

- Topics identified for the future included volumetric modulated arc therapy (VMAT), intensity modulated radiation therapy (IMRT), planning for electrons, craniospinal planning, ARIA administration and paediatrics.

Conclusion: The participants reported that infrastructure, budget and human resources would be the greatest challenges in implementing course knowledge in their own departments. The need for ongoing mentorship and skills development was identified. Development of an advanced techniques course will commence in 2019 , as well as shorter courses for the learning needs of South African teams.

\section{Aggressive end-of-life care versus palliative care interventions in patients with haematological malignancies: A social work perspective}

\section{A. De Beer \\ Private Practice, Johannesburg, South Africa}

The aim of this presentation is to provide attending social workers with an understanding of the difference between 
aggressive end-of-life care and palliative care interventions, including hospice referrals, in patients with haematological malignancies. The benefits and disadvantages of each approach will be explored. Particular attention will be paid to the ethical considerations of each approach. The patient's preferences as well as communication with the patient and their family regarding end-of-life care and withdrawing of treatment will be discussed.

\section{The outcome of patients with squamous cell carcinoma of the oropharynx treated with chemoradiotherapy at Groote Schuur Hospital over an 8-year period}

\author{
J.A. Wetter ${ }^{1,2}$ \\ ${ }^{1}$ Groote Schuur Hospital, Cape Town, South Africa \\ ${ }^{2}$ University of Cape Town, Cape Town, South Africa
}

Background: Definitive chemoradiotherapy is a standard treatment for squamous cell carcinoma (SCC) of the oropharynx; however, it is a regimen with many potential side effects - both acute and long-term. Even when patients have access to the best medical care, they frequently do not complete treatment as prescribed and/or are left with reduced function (swallowing, etc.) post-treatment. In theory, this rigorous regimen may be even less well tolerated in a socio-economic setting where patients have poorer baseline nutrition and more limited access to healthcare. These patients may present with more advanced disease and/or struggle to comply with scheduled treatments. Previous studies have shown that deviations from the chemoradiation protocol for head and neck SCCs compromise treatment outcome.

Objectives: The aims of this study are threefold: to determine the disease-free survival of patients with SCC of the oropharynx treated with definitive chemoradiotherapy in our hospital (a tertiary government hospital serving a large geographical area), to quantify the extent of protocol deviations within our system and to document the incidence of medium- and long-term side effects of this regimen in our patient population. These findings will guide us in deciding whether our treatment approach (in terms of patient selection, treatment prescribed, etc.) should be altered or may remain unchanged.

Method: This is a retrospective study in the form of a folder review of patients with SCC of the oropharynx treated at Groote Schuur Hospital between 2011 and 2018. All patients treated with definitive chemoradiotherapy (as opposed to surgery + / - adjuvant radiotherapy) were included. In almost all cases, chemoradiotherapy was preceded by neoadjuvant chemotherapy, mainly because of waiting times for radiotherapy. Staging was done as per AJCC version 7. Histological (biopsy) findings (HPV status, presence or absence of perineural invasion, grade of tumour, etc.) will be documented. The primary end-point of the study is the overall survival of patients with SCC of the oropharynx treated with definitive chemoradiotherapy. The secondary end-point of the study includes documenting the incidence of treatment protocol deviations as well as the incidence of medium- and long-term side effects of treatment.

Results: The folders of approximately 92-98 patients are in the process of being reviewed. This process is incomplete at this point. The documentation is complex as the information being collected is detailed and highly variable. An update of the results will follow as soon as all folders have been reviewed.

Conclusion: No final conclusions can be drawn at this time. The conclusions will follow once all results have been analysed. We believe that this study will give us a reasonable overview of how successful or unsuccessful our treatment of oropharyngeal SCC is in a limited resource setting. If the topic is accepted for presentation, a final update of this abstract will be forwarded to the organising committee prior to the congress.

\section{Descriptive epidemiological study of head and neck cancers at a single institution in Southern Africa}

\section{K. Naidoo}

Stellenbosch University, Cape Town, South Africa

Objectives: To describe the epidemiology of head and neck malignancies at Tygerberg Hospital (TBH) from 01 January 2015 to 31 December 2017. We evaluated patient demographics, risk factors, tumour characteristics, prognostic factors, disease stage, treatment intent and treatment modality.

Methods: Records of all head and neck cancer (HNC) patients who presented to Tygerberg Hospital's Oncology Department between 01 January 2015 and 31 December 2017 were reviewed. The following variables were described: patient demographics, which include age, sex, HIV status and socio-economic status, as well as tumour characteristics, risk factors, treatment intent and treatment modalities.

Results: Data were collected from 854 patients visited between 2015 and 2017. There were 603 (71\%) male and 251 (29\%) female patients. The male to female ratio was 2.4:1 and the age range was 10-89 years (median age 58 years). Smoking was a risk factor in $737(86.3 \%)$ and alcohol in 634 (74.2\%) patients. Eleven patients (2.22\%) were HPV-positive, of these $73.68 \%$ were in the oropharynx. The most common site was the oral cavity $(n=320)$ and the most common subsite was the anterior tongue $(n=137)$. Eleven patients had two separate primariesat the time of diagnosis. In total, 466 patients $(53.87 \%)$ presented with locally advanced, stage IVA disease.

Conclusions: The median age of diagnosis, the most predominate primary site, histological subtype and stage at presentation were consistent with that reported in the literature. The incidence of p16 positive oropharyngeal lesions differs greatly from the changing worldwide aetiological trends of squamous cell carcinoma (SCC) of the oropharynx. 


\section{Care, ethics and society: Supportive governance}

\section{R.P. Abratt ${ }^{1,2}$}

${ }^{1}$ Medical Specialist Holdings, Johannesburg, South Africa

${ }^{2}$ University of Cape Town, Cape Town, South Africa

Objectives: To review the role of Institutional Clinical Governance Committees (CGCs) as well as Social and Ethics Committees (SECs). The aims of these committees are to support professional performance, ethical behaviour and a cohesive society as well as to manage possible conflicts of interest and discrimination.

Method: The structures functioning and legal background of a CGC (established in 2015) and a SEC (established 2018) were reviewed.

Results: The CGC aims at high standards of care and quality improvement programmes. It is opportunity orientated. It functions in five domains: Clinical Excellence, Health Personnel, Evidence-Based Medicine, Environment (including facility accreditation) and Risk Management for both chemotherapy and radiotherapy. Objectives with measurable results are set on an annual basis for each of these domains. Peer-reviewed publications have resulted within all these domains since the inception of the CGC. In particular, an audit of user experience of the accreditation process showed that it was welcomed as being helpful in maintaining standards. The SEC aims to include societal interests and ethics in all decisions and actions. It sets a balanced framework for decisions and actions. It is regulated by the Company Act of 2008. These specify five domains of activity: Social and Economic Development, Good Corporate Citizenship, Environmental, Health and Safety, Consumer Relations and Labour and Employment.

Conclusion: The CGC is about performance in clinical care. The SEC is about a balanced framework for all decisions and actions. When successful, they will synergise high-quality clinical care and institutional and social well-being.

\section{Tuberculosis in children with cancer: Experience from a South African centre}

\section{G. Naidu ${ }^{1}$, A. Izu ${ }^{2}$, R. Wainwright ${ }^{1}$, S. Poyiadjis ${ }^{1}$, D. MacKinnon ${ }^{1}$, B. Rowe ${ }^{1}$ and SA Madhi ${ }^{2,3,4}$}

${ }^{1}$ Division of Paediatric Oncology, Department of Paediatrics, Chris Hani Baragwanath Hospital, University of the Witwatersrand, Johannesburg, South Africa

${ }^{2}$ Medical Research Council, Respiratory and Meningeal Pathogens Research Unit, Johannesburg, South Africa

${ }^{3}$ Departments of Science/National Research Foundation: Vaccine Preventable Diseases, Faculty of Health Sciences, University of the Witwatersrand, Johannesburg, South Africa

${ }^{4}$ National Institute for Communicable Diseases - a Division of National Health Laboratory Service, Sandringham, Johannesburg, South Africa

Background: Tuberculosis (TB) has been reported in children undergoing chemotherapy.
Objectives: The objective of this study was to:

- describe the incidence of latent TB infection and TB disease in children with cancer

- compare the Tuberculin Skin Test (TST) with the Interferon Gamma Release Assay (IGRA-T-SPOT.TB) for diagnosing latent TB infection

- describe the characteristics and outcome of children with TB disease and cancer .

Method: A prospective cohort study was conducted at a tertiary centre in South Africa from 01 April 2010 to 31 March 2012, where patients followed up until March 2015. Newly diagnosed children with cancer were enrolled and screened for latent TB infection with a TST and T-SPOT.TB test. Where TB disease was suspected during chemotherapy, sputum was sent for culture.

Results: A total of 169 patients were enrolled. Latent TB infection was documented in five patients $(2.9 \%)$. The T-SPOT. TB assay yielded negative results. Twenty-four (14.2\%) patients were diagnosed with TB disease during chemotherapy. The incidence of TB disease was 4.7 per 100 child years. Those that developed TB disease during chemotherapy had a higher number of high-dose corticosteroid courses (397.4 per 100 child years) compared to those who did not develop TB (40.7 per 100 child years; $p<0.001$ ). Children who developed TB disease had more septic episodes associated with profound neutropenia (319.5 vs. 46.6; $p<0.001$ ), profound lymphopenia (264.9 vs. $41 ; p<0.001)$ and profound monocytopenia (389.6 vs. 63.7; $p<0.001$ ). Children who developed TB had more septic episodes with prolonged neutropenia ( 350.6 vs. $51.1 ; p<0.001$ ), prolonged lymphopenia (335.1 vs. $59.2 ; p<0.001$ ) and prolonged monocytopenia (444.2 vs. 70.7; $p<0.001)$. There were $4 / 24(16.7 \%)$ deaths.

Conclusion: The incidence of latent $\mathrm{TB}$ infection in children with cancer was low. The TST and T-SPOT.TB tests have limited value in diagnosing TB-infection in immunocompromised patients. Risk factors for developing active TB disease in patients with haematological malignancies include the use of numerous courses of highdose corticosteroids and profound, prolonged neutropenia, lymphopenia and monocytopenia. Mortality in patients with $\mathrm{TB}$ and cancer is because of complex factors.

\section{Respiratory virus infections in children with febrile neutropenia: Experience from a South African centre}

\section{G.Naidu ${ }^{1}$, A. Izu², R. Wainwright ${ }^{1}$, S. Poyiadjis ${ }^{1}$, D. MacKinnon D ${ }^{1}$, B. Rowe ${ }^{1}$ and S.A. Madhi ${ }^{2,3,4}$}

${ }^{1}$ Division of Paediatric Oncology, Department of Paediatrics, Chris Hani Baragwanath Hospital, University of the Witwatersrand, Johannesburg, South Africa

${ }^{2}$ Medical Research Council: Respiratory and Meningeal Pathogens Research Unit, Johannesburg, South Africa

${ }^{3}$ Department of Science, National Research Foundation, Vaccine Preventable Diseases, Faculty of Health Sciences, University of the Witwatersrand, Johannesburg, South Africa 
${ }^{4}$ National Institute for Communicable Diseases - a Division of National Health Laboratory Service, Sandringham, Johannesburg, South Africa

Background: The role of respiratory viruses (RV) as infecting or co-infecting pathogens in patients with febrile neutropenia (FN) has not been extensively characterised.

Objective: The aim of this study was to:

- document the incidence of RV infections in children with haematologic malignancies and solid tumours, and to assess whether there was a difference between these groups

- identify the most common RVs occurring in children with FN

- document the incidence of RV infections in those children who died.

Methods: All children diagnosed with cancer between 01 April 2010 and 31 March 2012 and followed up until March 2015 at a paediatric oncology unit at a tertiary centre in South Africa were enrolled in a prospective cohort study. A suspected septic episode (SSE) was defined according to the standard definition of FN. During an SSE, blood investigations and nasopharyngeal aspirates were conducted and various parameters were recorded.

Results: There were 169 patients, 82 (48.5\%) with haematologic malignancies and $87(51.5 \%)$ with solid tumours. A total of 528 SSEs occurred. The number of SSEs associated with RV was $147 / 330(44.5 \%)$ in haematologic malignancies and 64/198 (32.3\%) in solid tumours ( $p=0.007)$. The number of SSEs with bacterial-viral co-infections was $112 / 330$ (33.9\%) in haematologic malignancies and 45/198 $(22.7 \%)$ in solid tumours $(p=0.01)$. The overall incidence per 100 child years of RV-associated SSEs was 61.2, with 92.4 in haematologic malignancies and 35.1 in solid tumours $(p<0.001)$. Human rhinovirus (HRV) was identified in 96/368 (26.1\%) of patients. Viral-viral co-infections occurred in $17.5 \%$ and RV-bacterial co-infection occurred in $35 \%$ of patients. Twelve children $(7.1 \%)$ died because of sepsis. Eighteen RVs were isolated in the 12 deceased patients: human coronavirus in $44.4 \%$ and HRV in $27.8 \%$.

Conclusion: Respiratory virus infections and bacterial-viral co-infections in SSEs were more common among children with haematologic malignancies compared to those with solid tumours. The most common RV identified was HRV. Viral-viral co-infections were lower than bacterial-viral co-infections. The patients who died had bacterial and fungal co-infections in addition to the identified RVs. The presence of RV-infection and its clinical significance and relationship with outcome remains uncertain.

\section{Immune reconstitution viral disease mimicking relapse with haematological malignancy}

\section{R. Thejpal, B.G. Neethling, Y. Goga, K. Coopasamy and H. Van Staaden}

Department of Paediatrics and Child Health, Nelson R Mandela School of Medicine, Inkosi Albert Luthuli Central Hospital, Johannesburg, South Africa
Background: Immune reconstitution inflammatory syndrome (IRIS) has been well documented in HIV-infected patients after anti-viral treatment has commenced. A similar phenomenon may occur in some patients with severe malnutrition when nutrition improves and in patients receiving chemotherapy with lymphocyte recovery.

Objective: The aim of this study was to document IRIS viral reticulo-endothelial hyperplasia in a series of HIV-negative patients suspected to have relapsed of malignancy.

Results: Three HIV-negative patients are described: two with CMV and one with EBV. Patient 1: a 3-year-old white male child with Burkitt lymphoma presented with extensive disease (orbital, abdominal, CNS and bone marrow involvement). He was treated with chemotherapy, including intra-thecal Rx and cranial radiotherapy. Mild tumour lysis occurred with treatment initiation. He achieved a complete remission and treatment was given over 9 months. Treatment was stopped after 3 months as he was noticed to have splenomegaly and lymphadenopathy (PET positive). Biopsy of an inguinal node showed CMV lymphadenitis. This resolved without further treatment. Patient 2: a 3-year 8-month-old Indian male child with B-precursor acute lymphoblastic leukaemia (ALL) presented with a white cell count of $34 \times 10^{9} / \mathrm{L}$, hepatosplenomegaly and CNS disease. He achieved complete remission with induction therapy and was given high-intensity blocks of chemotherapy. Maintenance chemotherapy (lower intensity $\mathrm{Rx}$ ) commenced 11 months after presentation. After 2 months relapse was suspected because of splenomegaly and small lymph nodes. Investigations for relapsed disease were negative. CMV viral load was $527828 / \mu \mathrm{L}$ ( $\log 5.7)$. Improvement occurred after treatment with valganciclovir and continuation of maintenance chemotherapy. Patient 3: a 4-year-old African male patient had empiric treatment for tuberculosis. Lymph node biopsy initially showed an EBV lympho-proliferative disorder. Six months later he developed increasing cervical adenopathy and dysphagia. Repeat biopsy showed a highgradeB-cell lymphoma. He received aggressivechemotherapy and went into clinical remission. After chemotherapy was stopped, increasing cervical nodes were noted and relapse suspected. Biopsy revealed a reactive lymph node with no malignant disease. This was possibly related to EBV and became smaller without specific treatment.

Conclusion: The above-mentioned patients demonstrated clinical features mimicking relapse of their original disease, which were because of viral infections that became apparent when chemotherapy was less intense or stopped.

\section{The significance of delays in radiotherapy}

\section{A.J. Hunter ${ }^{1,2}$ and A.S. Hendrikse ${ }^{1,2}$ \\ ${ }^{1}$ Radiobiology Section, Division of Radiation Oncology, University of Cape Town, Cape Town, South Africa \\ ${ }^{2}$ Groote Schuur Hospital, Cape Town, South Africa}

Objectives: The aim of this study was to review the evidence concerning the effect of radiotherapy treatment delays on tumour outcomes in a variety of common tumours. 
Method: A review of the recent literature was undertaken to identify articles that provided evidence concerning the effects of overall treatment time and time till treatment initiation in a selection of common tumour types. Where available, kick-off times and effective radiation doses lost because of inferred proliferation are reported.

Results: The effect of changes in overall treatment time and time to the initiation of radiotherapy has significant effects on certain tumour types, but results are highly variable. Introduction of modern therapies and combinations may influence the effect of treatment delays.

Conclusion: The results may provide guidance for radiobiological analysis and recommendations for handling of treatment delays. Heterogeneity of tumours and the development of modern therapies may influence the assumptions that should be used when attempting to estimate the effects of repopulation in a clinical setting.

\section{Cone beam computed tomography images used for dose calculation in adaptive radiotherapy at Netcare Olivedale Hospital}

\section{K.E. Dumela ${ }^{1}$, N.M. Tshishimbi ${ }^{2}$ and H. Harrison ${ }^{2}$}

${ }^{1}$ Netcare Medical Physics CoE, Netcare Ltd., Johannesburg, South Africa

${ }^{2}$ Netcare Olivedale Hospital, Netcare Ltd., Johannesburg, South Africa

Background: Cone beam computed tomography (CBCT) is an imaging device used for patient positioning and target localisation in real time. The use of daily CBCT allows the monitoring of patient weight loss, setup errors and tumour shrinkage during the course of radiotherapy treatment. One of the biggest challenges in radiotherapy is motion management. The patient target position variations arise from the setup error, online matching errors, changes in tumour position because of organ motion and by organ filling and changes in tumour sizes resulting in treating tumour or surrounding tissues inaccurately. Cone beam computed tomography images are used for dose calculation to determine the dosimetric deviation because of setup error, organ motion, tumour shrinkage and matching errors. These images are not used for patient treatment planning purposes.

Objectives: The aim of this study was to estimate the dosimetric deviation by using CBCT images for adaptive radiotherapy when the patient geometry changes and setup errors occur, and to determine feasibility, limitation concerns and usefulness of this method.

Method: The commissioning of the treatment planning system includes the CT calibration curve to convert the Hounsfield units (HUs) of different materials to electron density (ED) for accurate dose calculation. The calibration curve of the CT scanner is done using the ED phantom. The calibration of the HU to ED for CBCT images is done using Catphan 604 and ED phantom. The calibration curves for planning CT and CBCT obtained using ED curve and CBCT obtained using Catphan 604 were compared. To validate
CBCT dose distribution, a treatment plan using $6 \mathrm{MV}$ photon beam with a field size $10 \times 10 \mathrm{~cm}^{2}$ on pelvic phantom was generated for both $\mathrm{CBCT}$ and treatment planning $\mathrm{CT}$ images. The dose volume histogram (DVH) from both plans was compared. When the patient tumour shrinks during the course of the treatment, the $\mathrm{CBCT}$ images are used to calculate the dosimetric deviation between the original plan and the current plan with the change of tumour size. The DVH of both plans is evaluated and the deviation is calculated. And in case of incorrect setup this method is used to assess the actual treatment given.

Conclusion: The calibration curves for planning CT and CBCT were comparable. For tumour shrinkage patients with deviation greater than $3 \%$, re-scan using the planning CT will be done. Re-scanning and re-planning reduce the dose to the critical organs and give adequate dose to the tumour.

\section{Planning and treatment of a total skin electron irradiation patient: A case study}

\section{Potgieter and K. Laidlaw}

\section{Independent Clinical Oncology Network (ICON), Cape Town, South Africa}

Background: A 33-year-old male patient was diagnosed with mycosis fungoides. The patient was treated using a total skin electron irradiation (TSEI) technique and utilising the highdose-rate electrons that are available on the treatment unit.

Objectives: The aim of this case study was to give feedback on the planning and treatment of a TSEI treatment technique to aid in the treatment of mycosis fungoides patients in future.

Method: The patient was prescribed a dose of 36 Gy in 18 fractions. Before the commencement of treatment, a virtual simulation was performed on the patient to obtain the patient's diameter, length and the source-to-surface distance of the patient to the treatment unit. Using this information, the monitor units required to obtain the prescription were calculated, as well as the separation required between fields to facilitate a uniform dose distribution on the skin of the patient. During treatment, the patient was placed on a trolley below the treatment unit for easy and accurate setup of the patient for the different fields required for total body irradiation in a horizontal position. A $3 \mathrm{~mm}$ slab of Perspex was placed over the patient to meet the clinical requirements by attenuating the $6 \mathrm{MeV}$ beam to have a penetration equivalent to that of 4 $\mathrm{MeV}-5 \mathrm{MeV}$ mean electron energy. Diode measurements were performed in the first week of treatment. The patient's eyes, nails and genitals were shielded during the treatment using a combination of wax and lead. Before each treatment daily dose output checks were performed on the high-doserate energy to ensure accurate treatment.

Results: Using the TSEI technique the patient could be treated in the horizontal position with relative ease. The treatment time was significantly reduced by using the high-dose-rate electrons. The diode measurements that were performed in the first week of treatment showed good correlation to the 
dose that was planned. The daily output checks that were performed stayed well within the $2 \%$ tolerance limit as recommended by SASQART which provided confidence that the patient was treated accurately for each fraction.

Conclusion: This case demonstrates that the TSEI technique can be successfully implemented using high-dose-rate electrons for the treatment of patients with mycosis fungoides.

\section{Development, validation and first results of new comprehensive tool for digitally testing accuracy of multi-leaf collimators of megavoltage units}

\section{W.P.E. Boonzaier}

Equra Health, Cape Town, South Africa

Background: Because of the steep dose gradients and proximity of the organs at risk (OAR) to the target in radiotherapy, multileaf collimator (MLC) positional accuracy becomes an increasingly important feature for intensity modulated radiotherapy (IMRT)-type techniques. Previous studies have shown that systematic errors of as small as $0.5 \mathrm{~mm}$ can have significant dosimetric effects on the outcome of IMRT treatment delivery. In this study, the accuracy and versatility of a new digital MLC quality assurance tool were assessed.

Method: A new platform for testing MLC accuracy was developed using a picket fence test, using images from the online portal imaging device and in-house created software. Validation and acceptance testing for this method were performed, and the method was implemented as routine quality assurance to evaluate the accuracy of the MLC on Elekta and Siemens units within the company.

Results: EPID imaging could be used to measure a picket fence digitally. Software could be created to analyse the positional error with an average accuracy of $0.1 \mathrm{~mm}+0.1 \mathrm{~mm}$ and the width error with an accuracy of $0.1 \mathrm{~mm}+0.1 \mathrm{~mm}$ in the clinically applicable range. Additional testing could be performed to evaluate MLC accuracy at various gantry angles and during an arc delivery.

Conclusion: This quality assurance tool proved to be efficient enough for use on multiple platforms, yet sensitive enough to MLC errors and was implemented as a new inhouse quality assurance tool on a national level within Equra Health.

\section{Measured and calculated doses in the 'Claws'}

\section{C.J. Trauernicht ${ }^{1}$, E. Hering ${ }^{2}$, F. du Plessis ${ }^{3}$ and G. Maree ${ }^{4}$}

${ }^{1}$ Tygerberg Hospital, Stellenbosch University, Cape Town, South Africa

${ }^{2}$ University of Cape Town, Cape Town, South Africa

${ }^{3}$ University of the Free State, Bloemfontein, South Africa

${ }^{4}$ Groote Schuur Hospital, Cape Town, South Africa

Background: The 'Claws' is a gold applicator loaded with I-125 seeds, used as a temporary implant to treat retinoblastoma. Under general anaesthesia, a pericorneal ring is attached to the four extra-ocular muscles, and four appendages, each loaded with I-125 seeds, are inserted beneath the conjunctiva in-between each pair of muscles and attached anteriorly to the ring. The applicator has an inside diameter of $22 \mathrm{~mm}$. A typical treatment prescription is $40 \mathrm{~Gy}$ given over 4 days to the centre of the eye. General anaesthesia is also required for the removal of the applicator.

Method: Solid water eye phantoms were manufactured to allow for dose measurements in the 'Claws'. Dose measurements were done with thermoluminescent dosimeters (TLDs) and Gafchromic film. The four legs of the 'Claws' were each loaded with four seeds of the same activity and the phantom was irradiated.

Results: Dose points were added on the Gafchromic film during image analysis to coincide with the dimensions and positions of the TLDs during irradiation. Normalised dose distributions agreed remarkably well to within 1 standard deviation.

Conclusion: Normalised calculated doses showed discrepancies of more than $20 \%$ in certain areas of the 'Claws', with measured doses being lower than calculated doses. It is thus evident that the gold shielding of the applicator material is not taken into account adequately in the treatment planning system.

\section{Conformal radiation therapy for breast cancer}

\section{Sharma, F. Mahomed, L. Nethwadzi, S. Mbatha and D. Kometse}

Departments of Radiation Oncology and Medical Physics, Charlotte Maxeke Johannesburg Academic Hospital, University of the Witwatersrand, Johannesburg, South Africa

Objective: The aim of this study was to document the radiation doses to lung and heart (organs at risk) in addition to breast tumour volumes and to correlate with treatmentrelated toxicities.

Methods: A total of 115 patients with breast cancer underwent computed tomography (CT) scans and planning procedure after decision at the multidisciplinary management meeting during 2016-2017. Both breasts were almost equally affected (right - 62 patients [54\%] and left -53 patients [46\%]). All patients had MUGA scans before RT planning. Forty-three patients (37\%) had conservative breast surgery and 72 had mastectomies. Seventy-four patients $(64 \%)$ received $50 \mathrm{~Gy}$ in 25 fractions and 39 (34\%) patients had 40.05 Gy in 15 fractions. The radiation therapy was delivered using $6 \mathrm{Mv}$ photos with different fields. The patients with conservative surgery received a boost to the tumour bed following tangential field. The patients with locally advanced disease and nodal involvement received radiation to chest wall as well as lymph drainage areas. Forty-eight per cent (56 patients) had radiation to chest wall and lymph drainage areas; 30 patients (26\%) had tangential field and tumour bed boost.

Results: The lung constraints used were V20 = $\leq 30$ Gy, $\mathrm{V} 8=35 \%-40 \%$ and mean lung dose $=<15$ Gy. One hundred and six patients (92\%) achieved the lung constraints. 
The heart constraints used were V25 Gy $=<10 \%$ and mean heart dose $=<26 \mathrm{~Gy}$. The heart constraints could be achieved in all except one patient with respect to both V25 and mean heart dose.

Conclusion: Conformal radiation was able to achieve the aim of the study and constraints were maintained in over $90 \%$ patients. There is no toxicity reported up till now on follow-up.

\section{Low-grade gliomas treated at the University of Cape Town Academic Hospital Complex: 2001-2017}

\section{G. Kahl, A. Davidson, A. Figaji, K. Pillay, T. Kilborn, M. Hendricks, A. van Eyssen, J. Parkes and T. Naiker}

\section{University of Cape Town, Cape Town, South Africa}

Background: The majority of central nervous system tumours in children are low-grade gliomas (LGG). Long-term survival rates are high with a slow, progressive course. Children with LGG have a good overall prognosis, although tumour location and extent of resection affect outcome. Although complete tumour resection is the goal in the management of these tumours, this is not always possible. Adjuvant therapy thus has an important role.

Objectives: The aim of this study was to evaluate the characteristics of LGG in our setting, the role of adjuvant therapy including more targeted, novel biologic therapy such as BRAF/MEK inhibitors, and the outcome of children with LGG in our institution.

Method: A retrospective analysis was performed on all children diagnosed with LGG at Red Cross War Memorial Children's Hospital (RCWMCH) between 2001 and 2017. Data were collected from patient hospital folders, as well as Paediatric Oncology records and Groote Schuur Hospital Radiotherapy records.

Results: Eighty-five children (40 boys and 45 girls) aged 0.10-13.76 years (median age 4.74 years) were diagnosed with LGG between 2001 and 2017 at RCWMCH. Sixty-five patients $(76 \%)$ were WHO grade I, 18 patients were WHO grade II (21\%) and two patients' histology were indeterminate. Six patients had metastatic disease at presentation. The most common sites were the cerebellum $(27 \%)$, followed by the hypothalamus (17\%), optic nerves and cerebrum (11\% each). Forty-three patients had surgery upfront (51\%), of which 21 patients had a complete resection and 22 patients had debulking surgery. Thirty patients had a biopsy only (35\%), where definitive surgery was not feasible. Twenty-three patients $(27 \%)$ received chemotherapy. All but two patients were treated with vincristine/carboplatin upfront. The most common second-line agent was vinblastine. Twenty-two patients received radiotherapy (26\%), of which three patients progressed thereafter. The estimated 5-year overall survival (OS) was $86.8 \%$ and the estimated 5-year progression-free survival (PFS) was $42.8 \%$. BRAF status was determined in three patients, all of which were negative.
Conclusion: Our outcomes are similar to those achieved in developed countries. Chemotherapy and radiotherapy are valuable adjuncts to treatment. BRAF status should be tested where possible to determine whether biologic therapies can be used.

\section{Anaplastic ependymoma in early childhood}

\section{J. Riedemann}

\section{Groote Schuur Hospital, Cape Town, South Africa}

The incidence of paediatric intracranial ependymomas peaks in early childhood, with a median age of 5 years at diagnosis of and $30 \%-40 \%$ of patients less than 2 years old. Traditionally anaplastic ependymomas are histologically classified as grade III tumours; however, no association between grade and biologic aggressiveness has been established. Molecular subtyping has revealed distinct groups of tumours that differ in anatomical location, response to treatment and prognostic outcomes. Location is considered as a strong prognostic indicator, with a significant subset of supratentorial ependymomas containing a RELA fusion chromosomal rearrangement (ST-EPN-RELA), which potentiates oncogenic NF- $\kappa \mathrm{B}$ signalling and confers poorer outcomes. In addition, a subgroup of these tumours is YAP-fusion positive, especially in infants, and is associated with improved survival outcomes. In contrast, infratentorial anaplastic ependymomas are subdivided into posterior fossa group A (PF-EPN-A) and B (PF-EPN-B) tumours depending on genetic and methylation profiling. Multiple retrospective studies have now confirmed that gain of $1 \mathrm{q}$ is a predictive marker for increased local and distant disease progression. Loss of histone protein H3K27M because of aberrant methylation occurs in a significant subset of PF-EPN-A tumours and is of diagnostic relevance. PF-EPN-A tumours mostly occur in infants and young children, exhibit higher levels of genomic methylation, are more invasive with greater metastatic potential and carry worse prognosis. PF-EPN-B tumours generally occur in older children and young adults and may carry a more favourable prognosis than PF-EPN-A. It is now generally accepted that molecular subgrouping is the strongest predictor of progression-free and overall survival. As a means of clinical application, two case studies will be described. The first case is of a 3-year-old boy with ST-EPNRELA $^{+}$who underwent debulking surgery, followed by chemotherapy and craniospinal external beam radiotherapy (36.00 Gy/1.8 Gy $\times 20 \#$ to the craniospinal PTV using 3DCRT plus an $18.00 \mathrm{~Gy} / 1.8 \mathrm{~Gy} \times 10$ \# boost to the tumour bed PTV using VMAT). The second case is that of a 2-yearold girl with a H3K27M negative/1q EXO1 gain negative PF-EPN-A who underwent subtotal resection, followed by external beam radiotherapy $(54.00 \mathrm{~Gy} / 1.8 \mathrm{~Gy} \times 30$ \# to the PTV and 5.4 Gy/1.8 Gy $\times$ 3\# boost to the tumour bed using VMAT). The clinical pictures, respective management and short-term outcomes will be discussed. 
Women's sexuality post-gynaecological cancer treatment at Groote Schuur Hospital: A qualitative, descriptive study using a comprehensive framework

S.C. Pitcher ${ }^{1}$, N. Fakie ${ }^{1,2}$, T. Adams ${ }^{1,3}$, L. van Wijk ${ }^{1,2}$, R. Saidu ${ }^{1,3}$, L. Denny ${ }^{1,4}$ and J. Moodley ${ }^{1,5,6,7}$

${ }^{1}$ SAMRC Gynaecological Cancer Research Centre, University of Cape Town, Cape Town, South Africa

${ }^{2}$ Department of Radiation Oncology, Groote Schuur Hospital, Cape Town, South Africa

${ }^{3}$ Department of Gynaecological Oncology, Groote Schuur Hospital, Cape Town, South Africa

${ }^{4}$ Department of Obstetrics and Gynaecology, Groote Schuur Hospital, Cape Town, South Africa;

${ }^{5}$ Cancer Research Initiative, University of Cape Town, Cape Town, South Africa

${ }^{6}$ Women's Health Research Unit, University of Cape Town, Cape Town, South Africa

${ }^{7}$ School of Public Health and Family Medicine, University of Cape Town, Cape Town, South Africa

Objectives: This study aimed to investigate women's experiences of their sexuality post-gynaecological cancer treatment by using a comprehensive framework of sexuality, and to understand how their sexual health needs can best be addressed as part of cancer care.

Method: The study used a qualitative descriptive design. Participants were recruited through purposive sampling at follow-up clinics within Groote Schuur Hospital's Gynaecological Oncology Unit. The final sample consisted of 35 women aged 29-35 years. All women had been diagnosed with one or more gynaecological cancers and treated with surgery, chemotherapy, radiation or a combination of these. Data were collected using semi-structured, in-depth individual interviews in participants' home language. Pile sorting was used within the interviews to facilitate discussion about difficult topics. The data were analysed using thematic analysis.

Results: The results are expected to provide thorough insights into women's sexual functioning and psychosexual well-being post-treatment and how this affects their lives and relationships.

Conclusion: The study findings can help develop support programmes to improve patients' quality of life posttreatment. Furthermore, this research expands the qualitative literature relating to gynaecological cancers in South Africa.

\section{Groin lymph node status in operable vulva carcinoma among HIV-negative versus HIV-positive patients}

\section{A. Naidoo and G. Dreyer}

\section{University of Pretoria, Pretoria, South Africa}

Background: Vulvar cancer is 5.8 times higher in HIV and AIDS women compared with the general population. Positive metastatic groin lymph nodes (GLNs) represent advanced disease with increased surgical morbidity, with a more guarded prognosis and the requirement for postoperative adjuvant therapy. Not all metastatic lymph nodes are clinically palpable and not all clinically palpable lymph nodes are metastatic.

Objectives: The objective of this study was to describe GLN and tumour characteristics at initial presentation and on final histology post-surgery in HIV-negative (HivN) and HIV-positive (HivP) patients with operable vulva carcinoma.

Method: This was a retrospective clinical audit of patients with newly diagnosed operable vulva carcinoma, treated from 01 January 1999 to 31 December 2016 at Steve Biko Academic Hospital, University of Pretoria. Exclusions were patients with incomplete records, histology results indicative of benign disease and patients with other known gynaecologic cancers.

Results: Ninety-six patients were included, of which 46 were HivP; $88 \%$ were on ARVs and $92 \%$ had CD4 counts above 200 cells $/ \mathrm{mm}^{3}$. There were no major differences in the two groups for pre- and post-treatment tumour characteristics and histology, and clinical GLN findings with $81 \%$ being non-palpable bilaterally. HIV-positive patients, however, had increased GLN metastasis ( HivP $=51 \%$ vs. HivN $=23 \%$; $p=0.009)$. Non-palpable GLNs were more likely to be normal or reactive $(83 \%)$, with only $17 \%$ being metastatic. HIV-positive patients had significantly more reactive GLNs (HivP $=55 \%$ vs. HivN $=12 \% ; p<0.001$ ) and a non-significant trend towards more clinically occult metastases within this clinical finding ( $\mathrm{HivP}=23 \%$ vs. HivN $=12 \%$ ). The majority of clinically palpable GLNs were metastatic (77\%), while $23 \%$ were either normal or reactive on final histology. The majority of normal and reactive GLNs on final histology were actually non-palpable clinically ( $91 \%$ vs. $89 \%)$. Extra-capsular metastatic GLNs were palpable or suspicious clinically in $86 \%$. No difference was found for all these findings within the two groups. Metastatic GLNs were non-palpable in $42 \%$ with, however, a non-significant trend towards more clinically occult metastasis in HivP patients (HivP $=45 \%$ vs. $\mathrm{HivN}=38 \% ; p=0.435)$.

Conclusion: The HivP and HivN groups did not differ in clinical and histologic tumour features and clinical status of GLNs. HIV-positive patients had higher rates of GLN metastasis and a non-significant trend towards clinically occult metastasis.

\section{Positron emission tomography-computed tomography in the staging and planning of HIV-positive cervical cancer patients}

H.M. Simonds ${ }^{1}$, M.H. Botha ${ }^{1}$, A.E. Ellmann ${ }^{1}$, J. Warwick ${ }^{1}$, A. Doruyter ${ }^{1}$, F.H. Van der Merwe ${ }^{1}$ and J.S. Jacobson ${ }^{2}$

${ }^{1}$ Stellenbosch University, Cape Town, South Africa

${ }^{2}$ Columbia University, New York City, NY, United States of America

Background: Positron emission tomography-computed tomography (PET-CT) imaging is commonly used as a staging tool to identify nodal involvement in locally advanced cervical carcinoma (LACC), especially in developed countries 
with a low burden of disease. The value of PET-CT for staging HIV-infected patients with LACC has not been described. In South Africa, the age-standardised incidence rate of cervical carcinoma (CC) is 43.5/100 000 females, and the prevalence of HIV in the general population is $18.9 \%$. We analysed PET-CT findings in a cohort of patients with LACC in Cape Town, South Africa.

Methods: Participants had LACC FIGO Stage Ilb or IIIB, and were referred, on the basis of stage and the availability of bookings, for PET-CT/radiotherapy planning CT from January 2015 to December 2018. All patients with HIV were prescribed cART. A team of expert nuclear medicine physicians and radiologists reported the PET-CT examinations; these were then coded for nodal station involvement and distant disease. Descriptive statistics and chi-squared tests were used to compare patients with and without HIV.

Results: Of 1089 patients referred for radiotherapy (curative, adjuvant or palliative), 286 underwent PET-CT. Eighty-nine patients (31.2\%) were HIV-positive; their median CD4 cell count was 482 cells/ $\mu \mathrm{L}$ (IQR 295-608). PET-CT imaging found pelvic nodal involvement in 205 patients $(72.4 \%)$, including $77.3 \%$ of those who had HIV and $70.3 \%$ of those who did not $(p=0.22)$; para-aortic nodal disease in 114 patients (42.7\% of HIV+ vs. $38.8 \% \mathrm{HIV}-, p=0.53)$; and distant disease in 55 patients $(23.6 \%$ of HIV+ vs. $17.3 \%$ HIV-; $p=0.22)$. In total, 223 patients $(79.3 \%)$, including $81.8 \%$ of patients without and $75.0 \%$ of patients with HIV $(p=0.31)$, were prescribed standard fractionation EBRT. Twenty-two patients $(7.8 \%)$ were prescribed hypofractionated EBRT and 36 patients $(12.8 \%)$ palliative therapy. Five patients $(1.7 \%)$ did not return.

Conclusions: PET-CT imaging found no differences between LACC patients, with and without HIV, in nodal involvement or occult metastases and did not lead to, or justify, treatment differences.

\section{Evaluating palliative care training in the oncology registrar programme}

R. Krause ${ }^{1}$, J. Parkes ${ }^{1,2}$, D. Anderson', L. Hartman', A. Sherriff ${ }^{3}$ and L. Gwyther ${ }^{1}$

${ }^{1}$ University of Cape Town, Cape Town, South Africa

${ }^{2}$ College of Radiation Oncology, Cape Town, South Africa

${ }^{3}$ University of Free State, Bloemfontein, South Africa

Background: In 2016, the University of Cape Town together with the South African College of Radiation Oncology identified the need to integrate palliative care in the oncology curriculum at an intermediate level. In collaboration with CANSA, a 12-module curriculum was introduced at 5 teaching hospitals.

Objectives: The overall aim of this research was to evaluate the impact of such a 1-year palliative care (PC) course within the oncology registrar programme in South Africa. This was a prospective evaluation of an intervention using a mixedmode approach.
Method: Blended learning was developed to train and support students and facilitators across 5 universities. Evaluation forms were electronically collected to determine students' reactions to course materials. Pre- and post-MCQs were used to review knowledge. Focus group discussions were used to explore reactions, change in knowledge and skills and integration of PC. Case studies from oncology portfolios will be evaluated to determine the application and integration of skills in their oncology practice.

Results: The first objective of this research was to determine the reaction of oncology registrars and their supervisors to a PC course. There was an overwhelmingly positive reaction towards the course. Concerns about feasibility and appropriateness of the course were found unsubstantiated. The second objective was to determine change in knowledge and skills in PC. The MCQ demonstrated change in knowledge and skills in modules 7 and 8 (symptom management). The poor MCQ results are ascribed to poor question sequencing and variable response rates. The focus group discussions demonstrated change in knowledge and skills, especially communication skills and pain and symptom management. The focus groups indicated a change in approach to PC as an essential component of oncology. The third objective was to determine the application of knowledge and skills of PC in oncology practice. This was subjectively evaluated. Most registrars felt the course addressed topics that formed part of their daily work. Students were able to integrate the learnt principles of PC.

Conclusion: Palliative care forms part of daily practice of oncologists, and a structured curriculum enables clinicians to use an evidence-based approach. Supervisors of the oncology training programme and registrars are confident that the training of 12 modules across 1 year in early registrar time is feasible and appropriate. Teaching in communication skills and pain and symptom management were found to be the most valuable modules. All registrars felt that the skills learnt were applicable to daily oncology practice. In conclusion, this research demonstrated that PC training is an essential component of oncology training in the South African setting.

\section{Retrospective analysis of the therapeutic utility of capecitabine plus oxaliplatin in patients with colorectal cancer at Tygerberg Hospital}

\section{S. Kibudde and W. Begg}

Department of Clinical and Radiation Oncology, Tygerberg Hospital, Tygerberg Campus, Cape Town, South Africa

Objectives: The aim of this study was to retrospectively explore the efficacy and safety of capecitabine plus oxaliplatin in patients with colorectal cancer at Tygerberg Hospital between 2012 and 2017 .

Method: A review of medical records of adult patients with histological diagnosis of colorectal cancer at Tygerberg Hospital between June 2012 and June 2017 was conducted. Patients received oxaliplatin at a dose of $130 \mathrm{mg} / \mathrm{m}^{2}$ as an infusion over $2 \mathrm{~h}$ on day 1 and capecitabine was administered 
orally in two divided doses recommended at $12 \mathrm{~h}$ interval at a dose of $2000 \mathrm{mg} / \mathrm{m}^{2}$ on days 1-14 of every cycle, repeated every 3 weeks. For most patients, a computed tomography scan of the chest, abdomen and pelvis was indicated after the third or fourth cycle of chemotherapy to assess response. Objective response rates are reported and the rates of progression-free survival (PFS) at 12 and 24 months were estimated using the Kaplan-Meier methods and compared by the log-rank test using Stata version 13. A $p$-value of less than 0.05 was regarded as significant.

Results: A total of 63 participants were treated with capecitabine plus oxaliplatin (XELOX) regimen over the study period. The median age was 53 years, and $46 \%$ were women, with $60.3 \%$ having colon as primary site, and $95 \%$ had stage IV disease at the time of treatment with XELOX. Patients received an average of six cycles of chemotherapy. In $57.1 \%$ of participants, the intent of treatment was palliative. In the curative intent group, equal numbers of patients received XELOX as either neoadjuvant or adjuvant, and liver resection was performed in 20 patients $(31.8 \%$; 95\% CI: $0.21-0.45)$. Overall, the disease control rate was $88.9 \%$ (95\% CI: 0.78-0.95), with 13 patients attaining complete remission after initial treatment with XELOX. Approximately one-third of patients developed disease progression (30.4\%) and the 1-year PFS was $44.5 \%$ (95\% CI: $0.31-0.57$ ), while the 2-year PFS was $25.1 \%$ (95\% CI: 0.14-0.38). Regarding safety, thrombocytopenia was the most frequent adverse event (18.3\%) and overall 15.1\% patients experienced grades 3 and 4 toxicity.

Conclusion: Our findings confirm a good objective response rate with XELOX regimen and tolerable side effect profile regardless of the intent of treatment.

\section{Delays in the referral and primary management of cutaneous malignant melanoma at Tygerberg Hospital}

\section{S.N. Naidoo and H. Burger}

\section{Stellenbosch University, Cape Town, South Africa}

Background: Cutaneous malignant melanoma (CMM) is a major cause of skin cancer-related mortality. The time between diagnostic biopsy and surgical excision, or surgical interval (SI), is a modifiable factor that may impact melanoma outcomes. Delays in the SI are attributable to many factors unique to the population and the resource available. Although delays may evoke psychological stress in patients, the impact on morbidity and mortality is controversial and few guidelines exist which outline an optimal SI.

Objective: The aim of this study was to determine the SI in patients with resectable CMM.

Methods: A retrospective chart review was performed on patients referred to the Tygerberg Academic Hospital (TAH) Multidisciplinary Melanoma Clinic with histologically confirmed CMM between January 2015 and December 2017. Men and women $>18$ years with resectable melanoma
(T1b-T4b N0-3 M0-1a) who went on to receive definitive surgery were included in the review.

Results: The cohort of 40 patients comprising mostly white people referred from the Cape Metro Area, with a median age of 69.5 years at diagnosis. Most diagnostic biopsies $(67.5 \%)$ were performed by primary care clinicians in public and private health settings. Of the patients, $72.5 \%$ had T3 or T4 lesions and half underwent SLNB, which led to upstaging in $40 \%$ of cases. The median length of the SI was 13.6 weeks. Twenty-two patients (55\%) underwent excision within the recommended 12 weeks from diagnostic biopsy. The median time from diagnostic biopsy to multidisciplinary team meeting (MDT) was 4.8 weeks and from MDT to surgery it was 7.3 weeks. Statistical associations between patient, provider and tumour variables and the SI will be reported.

Conclusion: Surgical interval guidelines for cutaneous melanoma are poorly defined. Limited evidence suggests that definitive treatment should be offered timely, but does not need to be immediate. Quality indicators for the treatment of melanoma were developed in a number of countries and some aimed to include an SI. We have used 12 weeks as an extrapolation of international SI guidelines and even though this target was not met, the study is proposed to be of value in guiding future protocols and ultimately allowing $\mathrm{TBH}$ to provide an improved, timely service to patients with an already devastating disease.

\section{Patterns of practice of breast cancer irradiation in the Department of Radiation Oncology, Charlotte Maxeke Johannesburg Academic Hospital, 2010-2012}

\section{A.M.G. Bunga, F. Mahomed and V. Sharma}

\section{University of the Witwatersrand, Johannesburg, South Africa}

Background: Conducting survival analysis is important for a radiation oncology department to facilitate distribution of resources to conditions that benefit most from intervention. It also aids the institution in identifying potential areas for improvement.

Objectives: Comparative determination of local control, overall survival and type of disease progression relative to local, regional and international findings. The aim of this study was to ascertain factors that affect the overall survival of the local population.

Method: A retrospective review of patient records from January 2010 to December 2012 with 5-year follow-up was done. Ethical approvals were obtained. A proforma was used to collect data from patient files. A total of 95 patient files were identified and randomly selected for inclusion in the study. Data were entered into SPSS and analysed using descriptive statistics, Kaplan-Meier survival and Cox regression for bivariate analysis.

Results: Local recurrence rate was 4.2\%, 22.1\% had distant metastases and $50.5 \%$ of study population had no progression. The 5-year overall disease-free survival was $41.1 \%$; lost to 
follow-up rate was $23.2 \%$. Death rate was $30.5 \%$ over 5 -year follow-up. The median survival for progressed patients was 28 months. Factors found to be significant on univariate analysis were as follows: stage grouping $(p=0.047)$, radiation dose $(p=0.03)$, perinodal spread $(p=0.034)$ and number of involved nodes in women aged $\geq 50$ years $(p=0.021)$. Bivariate analysis also confirmed radiation dose and staging as significant factors $(p=0.023)$. Seventy-two per cent of those who received hypofractionated irradiation were still alive at 60 months of follow-up. Of the study population, $35.8 \%$ received radiotherapy within 8 weeks from previous intervention. Consideration for best-case and worst-case scenarios was conducted to account for loss-to-follow-up rates.

Conclusion: Hypofractionated radiotherapy has favourable outcomes in our setting. The loss-to-follow-up rate makes analysis reliable in descriptive statistics, but not reliable in survival analysis. Establishing a departmental database and regularly collecting information on patient outcomes to facilitate timely data analysis is recommended. There should be provision of incentive to patients for attending follow-up clinics to reduce loss-to-follow-up. Careful selection of patients to receive radiotherapy will aid in improving outcomes. Identifying patients needing radiotherapy at multidisciplinary team meetings to plan scheduling ahead of time is also essential.

\section{Clinician- and patient-reported outcomes for severe late bladder and gastrointestinal toxicity in locally advanced cervical cancer patients treated with chemoradiation}

\section{P. Pillay ${ }^{1}$, C. Minnaar ${ }^{1}$ and J. Kotzen ${ }^{2}$ \\ ${ }^{1}$ University of the Witwatersrand, Johannesburg, South Africa} ${ }^{2}$ Wits Donald Gordon Medical Centre, Johannesburg, South Africa

Objectives: The purpose of this study was to determine the incidence of severe late ( $\geq$ grade 3 and $>90$ days after treatment completion) bladder (BC) and gastrointestinal (GIC) complications and to describe the quality of life of these patients.

Method: This is a retrospective analysis of the control arm of a prospective phase-III randomised, controlled, trial (RCT) investigating addition of hyperthermia to chemoradiation from January 2014 to June 2017. A total of 76 participants (FIGO stage IIB-IIIB) were included. The Kaplan-Meier time to event analysis was used to determine actuarial probability of complications. Clinician-reported morbidity using RTOG criteria and patient-reported outcomes (PRO) using EORTC QLQ-CX24 Quality of Life (QoL) questionnaires were assessed at baseline, every 3 months post-treatment during the first year, and 6-monthly in the second year.

Results: The mean age was 50 years \pm 10.6 . Fifty per cent were HIV-positive and the median CD4 count was 518 (374-626). All participants completed the entire course of 50 Gy in 25 fractions of external beam radiation, three 8 Gy brachytherapy fractions and 1-2 doses of concurrent cisplatin within
56 days. Bladder complications are predominantly fistulas, which was highest in the first year and half as likely in those with no evidence of disease (NED). Gastrointestinal complications were largely obstructive, predominantly in the second year with a similar risk irrespective of disease presence. In those with NED, 5.3\% developed $\geq$ grade 3 BC (grade 5 fatality $=1$ ) with an actuarial probability of $3.3 \%$ and $12.5 \%$ at first and second years, respectively; $3.5 \%$ developed $\geq$ grade 3 GIC (grade 5 fatality $=2$ ) with $0 \%$ at first year and $23.1 \%$ actuarial risk at second year. The probability of being alive with or without complications was not statistically different (chi-square 0.788, $p=0.375$; log rank: Mantel-Cox). However, those without complications lived longer and the median survival was not yet reached. Patients with complications reported more severe symptoms (bladder $20 \%$ vs. $5 \%$ and GIT $21 \%$ vs. $8 \%$ ) and these tend to be complicationspecific symptoms, for example, incontinence in fistulas or related obstructive symptoms. There was a discordance between clinician graded and PRO (bladder $9.2 \%$ vs. $7 \%$ and GIT $7.9 \%$ vs. $10 \%$ ). Clinicians grading using the worst toxicity underestimated the time and quality of life that participants endure with grades 3 and 4 symptoms before a grade 5 fatality.

Conclusion: There is a high risk of developing severe complications in patients who survive the first year, and their QoL outlook is significantly worse than those without complications. Patterns of telltale symptoms and their intensity reported by patients are our best early warning system to detect severe late complications.

\section{Using a two-phase electron radiotherapy technique to manage early-stage Dupuytren's disease: Outcomes of a case series}

\author{
O. Obuseng ${ }^{1,2}$, T. Naiker ${ }^{1,2}$, M. Solomons ${ }^{1,2}$ and J. Parkes ${ }^{1,2}$ \\ ${ }^{1}$ University of CapeTown, Cape Town, South Africa \\ ${ }^{2}$ Groote Schuur Hospital, Cape Town, South Africa
}

Objectives: Prophylactic irradiation, orthovoltage and electron therapy have shown benefits in early-stage Dupuytren's disease (DD). Much of the data, however, is derived from retrospective clinical trials with variable patient, treatment and disease parameters, making it difficult to draw definitive conclusions. In a prospective randomised controlled trial (RCT) comparing low- and medium-dose radiotherapy (RT) to observation, long-term results demonstrated that RT reduced progression and the need for hand surgery more than 5 years post-treatment, with acceptable acute and late effects. We present the outcomes of five cases with early-stage disease treated with medium-dose electron therapy protocol in our institution.

Method: This study was designed as a retrospective case series of patients referred to Groote Schuur Hospital (GSH) for RT to prevent disease progression. After clinical assessment, patients were staged according to a modified classification of Tubiana et al. and only patients with earlystage disease ( $\mathrm{N}$ and $\mathrm{N} / \mathrm{I}$ ) were considered eligible for the study. Five patients (nine hands) were included in the final 
analysis. Once informed consent was obtained, patients were irradiated with $4 \mathrm{meV}$ electrons between February 2013 and April 2018, according to a protocol developed in Heidelberg, Germany. Treatment was administered in two phases of $15 \mathrm{~Gy}$ each to a total of $30 \mathrm{~Gy}$, delivered as 5 fractions of 3 Gy each on five consecutive days followed by a 6-12-week break before delivery of the second phase of treatment. Preand post-treatment parameters, both subjective (symptoms and patient satisfaction) and objective (numbers and sizes of nodules and cords and comparative pictures), were compared to assess treatment response. The patients were also asked to report all acute and late side effects of treatment.

Results: Final results pending.

Conclusion: At the time of abstract submission, some patient responses and images were still outstanding. It is expected that these will be received within the coming weeks. Once received, results will be analysed and the abstract will be finalised for final submission to the South African Congress of Oncology (SACO) Abstract Committee for consideration.

\section{Cytogenetics in B-lineage acute lymphoblastic leukaemia: Translocation $t(1 ; 19)$ (q23;p13.3) E2A-PBX1 is a poor prognostic criterion in children of African descent}

\section{J.E. Poole ${ }^{1}$, N. Bouwer ${ }^{2}$, T. Wiggell ${ }^{2}$ and E. Benade ${ }^{2}$}

${ }^{1}$ Charlotte Maxeke Johannesburg Academic Hospital, Faculty of Health Sciences, University of the Witwatersrand, Johannesburg, South Africa

${ }^{2}$ Department of Molecular Medicine and Haematology, National Health Laboratory Service, Sandringham, Johannesburg, South Africa

Background: Cytogenetics is used to stratify B-linage acute lymphoblastic leukaemia (ALL) into prognostic risk groups. Hyperdiploidy and translocation $t(12 ; 21)$ indicate a favourable prognosis, whereas translocation $t(9 ; 22)$ and MLL gene rearrangements indicate an unfavourable prognosis. Translocation $\mathrm{t}(1 ; 19)$ E2A-PBX1 has not been used in the major clinical trials in high-income counties (HIC) as an unfavourable prognostic factor, and the literature is not conclusive.

Method: A retrospective chart review was performed on all ALL patients treated at the Charlotte Maxeke Johannesburg Academic Hospital, and Wits Donald Gordon Medical Centre, Johannesburg, South Africa, from 2002 to 2018. Initial bone marrow examination included immunophenotyping by flow cytometry, cytogenetics, ploidy by flow cytometry and fluorescent in situ hybridisation (FISH) for the ALL quadruple FISH.

Results: A total of 322 patients were diagnosed with ALL. Of these, 241 (74.7\%) were diagnosed with B-linage ALL. There were 224 with early Pre-B/CALL, 11 with pro-B/Null ALL and 5 patients who also had mature $B$ antigen expression with surface membrane immunoglobulin and CD20. Thirtysix patients $(16 \%)$ tested positive for $t(1 ; 19)$. All five patients with mature B elements were $t(1 ; 19)$-positive. The patients with $t(1 ; 19)$ had a mean age of 7.18 years, a male-to-female ratio of $0.6: 1$ and a mean white cell count of $97.8 \times 10^{9} / \mathrm{L}$. Thirty patients $(83 \%)$ were of black African descent. Cytogenetics of this group showed a complex karyotype in 17 of the 36 patients $(47 \%)$ in addition to the translocation $t(1: 19)$. The EFS of the $t(1 ; 19)$ group was $55 \%$, as opposed to $90 \%$ in the $t(12 ; 21)$ group, and $80 \%$ in the patients with no particular FISH abnormality.

Conclusion: Patients with translocation $t(1 ; 19)$ present in the older child, have a higher white cell count at diagnosis and occur in patients of African descent. The inferior survival rate suggests a poorer prognosis, and that $t(1 ; 19)$ should be used in the risk stratification of B-lineage ALL.

\section{Paediatric anaplastic large-cell lymphoma: A single unit experience in KwaZulu-Natal, South Africa}

\section{Y. Goga ${ }^{1,2}$, R. Thejpal ${ }^{1,2}$, B.G. Neethling ${ }^{1,2}$, K. Coopasamy ${ }^{1,2}$ and H.B. Van Staden ${ }^{1,2}$}

${ }^{1}$ Inkosi Albert Luthuli Central Hospital, Nelson R Mandela School of Medicine, University of KwaZulu-Natal, Durban, South Africa

${ }^{2}$ Paediatric Haematology Oncology Unit, Department of

Paediatrics and Child Health, Durban, South Africa

Background: Anaplastic large-cell lymphoma (ALCL) accounts for $10 \%$ of paediatric lymphoma. In areas with a high tuberculosis (TB) burden, lymphoma is often misdiagnosed as TB and diagnosis is delayed.

Objectives: We sought to describe patient characteristics, treatment response and survival of patients diagnosed with ALCL at a single centre from January 2003 to December 2017.

Method: A retrospective review of patients (aged 12 and under) diagnosed with ALCL from January 2003 to December 2017 presenting to the Paediatric Unit at Inkosi Albert Luthuli Central Hospital was done.

Results: Eighteen patients were diagnosed over a 15-year period. The male to female distribution was 11:7. All 18 patients were black people. Patients' age range was 2-12 years. Two patients were HIV-positive. There were two patients each with stages 2 and 3 disease, and 14/18 patients had stage 4 disease. Half of the patients with stages 2 and 3 disease are alive, and the other half have been lost to follow-up (LTFU). Patients were treated with the NHL-BFM 90 protocol. Of the 14 patients with stage 4 disease, three are alive (survival 21 months -5 years), one LTFU, eight died from primary/progressive/early relapsed disease and two were late deaths (one relapse and one second malignancy). Of the two HIV-positive patients, one was LTFU after 6 months and the second one died after developing acute biphenotypic leukaemia after 3 years. ALK/t2,5 status was positive in eight patients (six alive), negative in five patients (four dead and one LTFU) and unknown in five patients (four dead and one LTFU). Six patients with stage 4 disease were treated for TB between 2 and 7 months prior to diagnosis, and in another five patients there were delays of between 1 and 10 months. Stage 4 disease presented with a wide range of signs, including 
pleural effusions, tender lymphadenopathy, bone lesions, gibbus, ascites and significant abdominal adenopathy.

Conclusion: Most of the patients had stage 4 disease with poor outcomes because of delays in diagnosis and incorrectly being treated for TB. Intensive public awareness on early warning signs and further clinician education enabling earlier diagnosis are needed to improve survival.

\section{Single-centre experience of B-cell lymphomas in children at the height of the HIV epidemic in South Africa}

\section{G. Kahl and A. Davidson}

University of Cape Town, Cape Town, South Africa

Background: South Africa has seen an increase in B-cell lymphomas (BCL) associated with HIV and AIDS. Burkitt lymphoma (BL), the most common subtype, occurs mostly in the sporadic form in South Africa.

Objectives: The purpose of this study was to evaluate the outcome of children with BCL, with and without HIV infection, assess the most common histology and determine whether the HIV epidemic has increased the incidence of BCL.

Method: The study was a retrospective folder review of patients with BCL at Red Cross Children's Hospital, Cape Town, between January 2003 and December 2015.

Results: Ninety-eight patients were diagnosed with BCL with a median age of 6.41 years. Sixty-nine patients were HIV-negative and 29 patients were HIV-positive. The annual incidence of BCL in the HIV-negative group was 4.3 versus 5.3 cases per year for 1991-2002 and 2003-2015, respectively. In the latter period, 2.2 additional cases of BCL were diagnosed in HIV-positive children. In the HIV-positive group, 19 (66\%) had BL, seven (24\%) had diffuse large B-cell lymphoma (DLBCL) and three (10\%) had other subtypes of BCL. Twelve (41\%) patients were on antiretroviral therapy at diagnosis. Seven HIV-positive patients were not treated with chemotherapy because of poor performance status, parental refusal or post-mortem diagnosis. In the HIV-positive group, one patient was in LMB risk group A (3\%), 19 patients in group B $(65 \%)$ and eight patients in group C $(28 \%)$. One patient had a primary CNS lymphoma. In the HIV-negative group, eight patients were in group A (12\%), 38 patients in group B (55\%) and 23 patients in group C (33\%). Overall, 5-year event-free survival was $81 \%$; specifically, HIV-negative group: $84 \%(n=69)$ and HIV-positive group: $70 \%(n=22)$. Survival rates were not significantly different between the HIV-positive and negative groups for groups B and C $(p=0.20$ and 0.37 , respectively).

Conclusion: The outcomes for HIV-positive children with BCL were not significantly worse than the survival of HIV-negative children in the same time period. Although BL was the most common subtype overall for both the HIV-positive and negative groups, DLBCL was proportionally more common in the HIV-positive group. The HIV epidemic has produced a modest increase in the incidence of BCL.

\section{Rare relapsed/second non-Hodgkin lymphoma in an HIV-infected child}

\section{R. Thejpal, B.G. Neethling, Y. Goga, K. Coopasamy and H. van Staaden}

Department of Paediatrics and Child Health, Nelson R Mandela School of Medicine, Inkosi Albert Luthuli Central Hospital, Durban, South Africa

Background: HIV infection is associated with an increased risk of malignancy, which is reduced with anti-retroviral treatment (ARVs). Children with HIV have a higher incidence of non-Hodgkin lymphoma (NHL) and Kaposi sarcoma.

Objectives: The aim of this study was to describe an ARV naive child who presented with spinal NHL and who developed a second NHL involving the pericardium.

Results: A 3-year-old African male child presented with acute flaccid paralysis. Initial CSF showed no cells, normal glucose and protein of $5.46 \mathrm{~g} / \mathrm{L}$. He was treated for a Guillain-Barre syndrome with i.v. immunoglobulin, with no effect. Radio-imaging showed axillary and abdominal lymphadenopathy and hypoechoic lesions in the spleen. HIV infection was confirmed with the initial viral load of $69243 / \mu \mathrm{L}$ and CD4 count of 890 (19.5\%). Antiretrovirals were commenced. A lymph node biopsy showed changes consistent with HIV infection. A subsequent MRI of the spine showed an extra-dural mass within the spinal canal extending from $\mathrm{C} 7$ to $\mathrm{T} 6 / \mathrm{T} 7$ displacing and compressing the spinal cord and extending into several neural foramina. Laminectomy and decompression of the spinal cord were performed 7 weeks after presentation and biopsy showed a high-grade NHL with features of a Burkitt lymphoma (BCL6, CD10 and EBER positivity with a high Ki67 proliferation index and a C-MYC translocation $\mathrm{t}(8 ; 14))$. He completed treatment for Burkitt lymphoma over the following 8 months. Towards the end of treatment, the patient showed some improvement in the paraparesis. This continued during the follow-up period while he was off chemotherapy and on ARVs. He remained well until he presented with recurrent pericardial effusions 53 months after stopping chemotherapy. His viral load was undetectable and the CD4 count normal. Typing of the malignant cells showed B lymphocytes with kappa restriction and CD19, CD20, CD79b and CD5 positivity. CD10 was negative and cytogenetic studies for $\mathrm{t}(8 ; 14)$ were negative. The long period of remission and the phenotype strongly support a second NHL. He is currently receiving chemotherapy.

Conclusion: The initial presentation as acute flaccid paralysis and primary spinal NHL is rare. The second presentation with pericardial lymphoma on ARVs with viral suppression with a likely second high-grade lymphoma has not been reported in HIV-infected children. 


\section{A cost comparison of rasburicase versus dialysis in the management of children with acute leukaemia at a South African centre}

\section{Kahts, A. Davidson, A. van Eyssen, P. Nourse, M. McCulloch and M. Hendricks}

\section{University of Cape Town, Cape Town, South Africa}

Background: Tumour lysis syndrome (TLS) is a common complication of haematological malignancies and has historically been managed with hyperhydration, urine alkalinisation and allopurinol with renal dialysis reserved for patients in acute renal failure. Rasburicase has been shown to drastically reduce the need for dialysis; however, its use is limited in developing countries because of its cost and availability.

Objectives: This retrospective analysis aimed to compare the cost to state per patient of rasburicase compared to dialysis in the management and prevention of tumour lysis syndrome in paediatric patients presenting with haematological malignancies admitted to Red Cross War Memorial Children's Hospital (RCWMCH).

Method: This study is a retrospective analysis of administrative data of paediatric patients with haematological malignancies who required dialysis or rasburicase in the management or prevention of TLS since the availability of rasburicase at RCWMCH in September 2014. Patients from two consecutive 35-month periods, before and after the availability of rasburicase at $\mathrm{RCWMCH}$, were grouped according to treatment modality, and the cumulative costs of hospitalisation, dialysis and drug administration were compared.

Results: The groups were comparable in mean age and gender. The mean total length of hospital stay was 10.04 days shorter for the rasburicase group than the dialysis group, with the average cost per patient in the rasburicase group being R40 989.64 lower than the dialysis group.

Conclusion: The use of rasburicase results in a significant per patient cost saving when compared to dialysis, which often requires intensive care admission and results in extended hospitalisation. The study supports the continued use of rasburicase as an essential adjunct in the management and prevention of TLS, reaffirming its use as a cost-effective and efficient drug.

\section{Preoperative CA 125 levels accurately predict lymphatic metastases among South African endometrium cancer patients at high risk for late stage}

\section{K. Hapsari, C. Visser, J. Makin and G. Dreyer}

\section{Universityof Pretoria, Pretoria, South Africa}

Objectives: The purpose of this study was to evaluate the predictive value of serum CA 125 levels in the pre-operative assessment of endometrium carcinoma in a setting where late presentation is common.
Method: Data were collected retrospectively from patient files from January 2012 to January 2017 at the Gynaecological Oncology Unit, Steve Biko Academic Hospital. All patients with pathologically proven endometrial carcinoma, scheduled for surgery, who had pre-operative serum CA 125 test results, were included in the study. The association of CA 125 with various histological factors was determined to evaluate sensitivity and specificity using Spearman correlation and receiver-operating characteristic (ROC) curves.

Results: A total of 58 endometrial cancer patients with complete records were included in the study. The mean age was 63 years (standard deviation [s.d.] 8.80; range 41-84 years). Postmenopausal bleeding was reported in $94.6 \%$ of patients. All patients underwent surgical staging, total abdominal hysterectomy with bilateral salpingooophorectomy and pelvic and/or para-aortic lymph node dissection. The number of lymph nodes removed ranged between 1 and 46 (median: 10 nodes; s.d. 8.96), while preoperative CA-125 serum values ranged from $3 \mathrm{IU} / \mathrm{mL}$ to $400 \mathrm{IU} / \mathrm{mL}$ (median: $21 \mathrm{IU} / \mathrm{mL}$; s.d. 85.4). The FIGO stage distribution was $41.4 \%, 15.5 \%, 36.2 \%$ and $6.9 \%$ (FIGO stages I, II, III and IV, respectively). No significant correlation was found of CA 125 with histopathological type ( $p=0.257)$, but there was a significant correlation with grade $(p<0.05)$, advanced FIGO stage $(p<0.001)$, depth of invasion $(p<0.001)$ as well as lymph nodes metastases $(p<0.001)$. In extra uterine metastasis, CA 125 values were found to be statistically significant different for lymph nodes metastasis $(p<0.001)$, cervical $(p<0.05)$ and ovarian involvement $(p<0.05)$. The most appropriate cut-off point of CA 125, where an increase in sensitivity was not associated with a fall-off in specificity, was $20 \mathrm{IU} / \mathrm{mL}$, reaching a sensitivity of $90 \%$ and a specificity of $67 \%$ for detection of lymph node metastases.

Conclusion: In our cohort of endometrial cancer patients, the preoperative serum CA 125 level was associated with lymph node metastases; we found a CA 125 of $20 \mathrm{IU} / \mathrm{mL}$ or more to be predictive. These findings suggest that among similar populations, CA 125 could be done preoperatively and could be used to determine the need for node dissection. This finding should, however, be validated in a larger prospective study on early-stage disease.

\section{A seamless solution for image-guided adaptive radiotherapy verification and dose summation}

\section{W. Shaw ${ }^{1,2}$, L.J. Strauss ${ }^{1,2}$ and A. Van Eck ${ }^{1,2}$ \\ ${ }^{1}$ University of the Free State, Bloemfontein, South Africa 'Inverse Square, South Africa}

Objectives: An online software application with real-time component tracking and dose calculation in linear accelerator treatment was developed for total dose computation in the verification of image-guided adaptive radiotherapy (IGART). The application can also be utilised for the treatment verification and quality assurance.

Method: Intensity-modulated radiotherapy techniques, such as volumetric modulated arc therapy (VMAT), require 
significant investment in online and offline treatment verification devices. Dosimetric verification requires substantial measurement efforts, human resources, nonstandard working hours and overtime efforts, as well as routine device setup and dismantling. In high-volume treatment centres, these requirements could limit the number of VMAT plans generated, let alone the option of plan adaptation for patients who may benefit from it. We have developed an online software application that automatically tracks each treatment fraction delivered by a linear accelerator, patient-related details and creates a log of linac component statuses during treatment. Upon treatment completion, a DICOM-RT plan is generated for re-calculation of the treatment dose based on the logged data. The application was extensively tested against several types of treatment interruptions, monitoring of components during beam on and off, as well as several repeated treatment test cases. Dose recalculation was tested on the SciMoCa Monte Carlo dose engine.

Results: The software application is easy to use and does not require any user interventions during operation. The application 'fishes' for data continuously and automatically bins the data into logged data files with patient ID association. Application testing in this regard gave similar results on Elekta Synergy, Precise (South Africa) and Versa HD linacs (Germany and Austria, respectively). Treatment logs and DICOM-RT plans were generated successfully for every treatment fraction. DICOM-RT files can be generated containing each logged entry or each planned control point. Negligible differences were found when comparing sequential treatment fractions in highly modulated VMAT beams, although any difference in linac component values is considered during dose calculation. Dose summation using biological indices allows tracking of the dose during adaptive treatment execution.

Conclusion: A novel software application was developed for linac component logging to generate full or filtered treatment logs. Automatic DICOM conversion allows dose recalculation serving as a high-impact Quality Assurance module. The ease of use and speed of calculation permit extension to IGART dose tracking.

\section{Review on hyperthermia techniques for the management of cervical cancer and the treatment outcomes}

\section{C.A. Minnaar ${ }^{1}$ and J.A. Kotzen ${ }^{1,2}$}

${ }^{1}$ University of the Witwatersrand, Johannesburg, South Africa

${ }^{2}$ Wits Donald Gordon Medical Centre, Johannesburg, South Africa

Background: Several hyperthermia (HT) techniques have been developed and investigated over the last two decades.

Objectives: We review the trials, including our local trial, and report on outcomes based on protocols and techniques used to treat cervical cancer.

Method: A literature search on PubMed was conducted using the key words 'hyperthermia', 'thermotherapy' and 'cervical cancer'. Trials were grouped according to treatment regimens: HT + radiotherapy (RTHT), HT + chemoradiotherapy (CRTHT) and HT + chemotherapy (CHT). The HT protocols, techniques and outcomes were tabulated.

Results: Two heating techniques are used: capacitive coupling heating using either $8 \mathrm{MHz}$ radiofrequency (RF) at $1200 \mathrm{~W}$ or $13.56 \mathrm{MHz} \mathrm{RF}$ at $150 \mathrm{~W}$, or radiative heating. Differences in protocols, technical specifications and administration exist between various techniques. Radiative heating requires shielding and requires patients to be sedated. Phase III trials using each technique have demonstrated a local control benefit (capacitive heating: $80 \%$ vs. $50 \%$ in the control arm, $p=0.048$; radiative heating: $83 \%$ vs. $57 \%$ in the control arm, $p=0.003$ ) and survival benefit (capacitive heating: $80 \%$ vs. $49 \%$ 3-year relapse-free survival in the control group, $p=0.048$; radiative heating: $51 \%$ vs. $27 \% 3$-year survival in the control arm, $p=0.009$ ) with the addition of HT to radiotherapy. Phases I and II studies on CRTHT using both techniques showed acceptable toxicities. Two phase III trials on CRTHT (one on each technique) applied HT once per week and did not show any benefit. A third phase III trial is in progress in Johannesburg using $13.56 \mathrm{MHz}$ RF capacitive coupled heating (power $130 \mathrm{~W}$ ) applied twice per week. Local disease control at 6 months is significantly improved ( $46 \%$ vs. $24 \%, p=0.003$ ) and preliminary results on 3-year survival show significant improvement (58\% vs. $35 \%$, $p=0.042$ ) with the addition of HT. Studies have shown safety and improved local control using CHT for the management of local recurrences or residual disease in previously irradiated cervical cancer patients (phase II study on radiative heating: objective response $=55 \%$; randomised study on capacitive coupled $13.56 \mathrm{MHz}$ RF heating: overall response rate $=72 \%$ vs. $35 \%$ in the control group, $p=0.0461$ )

Conclusion: Hyperthermia is an effective radiosensitiser for cervical cancer patients who cannot receive chemotherapy. When applied twice a week to CRT protocols using capacitive coupled $13.56 \mathrm{MHz}$ RF, HT improves the local disease response and may improve the 3-year disease-free survival. Chemotherapy may improve local control in patients with recurrent or residual disease in a previously irradiated region where treatment options are limited.

\section{Use of palliative chemotherapy in South Africa: National survey of paediatric oncologists}

\section{A. Büchner ${ }^{1}$, A. Davidson ${ }^{2}$ and M.A. Meiring ${ }^{3,4}$}

${ }^{1}$ Paediatric Haematology Oncology, Department of Paediatrics, Steve Biko Academic Hospital, University of Pretoria, Pretoria, South Africa

${ }^{2}$ Haematology Oncology Service, Department of Paediatrics and Child Health, Red Cross War Memorial Children's Hospital,

University of Cape Town, Cape Town, South Africa

${ }^{3}$ Universityof Cape Town, Cape Town, South Africa

${ }^{4}$ Paedspal, Cape Town, South Africa

Background: Palliative chemotherapy is a cancer-directed therapy that aims at stopping or slowing down the progression of the malignancy even though it may not have any potential for remission or cure. There are different 
reasons why cancer in children may be advanced and even incurable at presentation to an oncology unit. In these situations, palliative chemotherapy may be offered in an attempt to improve the quality of life and prolong survival.

Objectives: This research study evaluated South African paediatric oncologists' perspectives and practices regarding the use of chemotherapy in patients with cancer with no realistic hope of cure.

Method: An online survey was conducted among paediatric oncologists in South Africa. The main aims of the questionnaire were to assess paediatric oncologists' considerations around the use of palliative chemotherapy, and then focus on the most recent patients treated with palliative chemotherapy. A total of 41 participants completed the survey, giving a response rate of $89 \%$.

Results: The majority of the paediatric oncologists were either neutral or agreed that palliative chemotherapy should be considered. The most important treatment aim was to improve quality of life of the patient $(92.7 \%$ of respondents agreed that palliative chemotherapy should be given to maximise the quality of life). The most important considerations when prescribing palliative chemotherapy were to minimise the toxicity of chemotherapy regimen (4.56 mean, standard deviation [s.d.] $=0.5$ utilising a 5 -point scale where $1=$ not important to $5=$ very important), and the effectiveness of the chemotherapy $(4.37$; s.d. $=0.48)$. Parental preference was not reported to be one of the most important factors when deciding on palliative chemotherapy and only $19.5 \%$ of patients received treatment primarily because it was requested by parents. The most important treatment considerations were largely achieved as expected in the most recent patient treated with palliative chemotherapy. Individual opinions and preferences with regard to recommending palliative chemotherapy differ between paediatric oncologists.

Conclusion: Although the overall attitude towards the use of palliative chemotherapy is positive, there is great inter-individual variation between oncologists in opinions and experience. The lack of empirical data to justify recommendations with regard to palliative chemotherapy remains a problem, and the researchers believe that this study will spark productive discussion and planning towards more structured use of palliative chemotherapy in children with cancer in South Africa.

\section{Use of palliative chemotherapy in South Africa: Experience in a single unit}

\section{A. Büchner ${ }^{1}$, A. Davidson ${ }^{2}$, D.T. Reynders ${ }^{1}$, F.E. Omar ${ }^{1}$, T.U. Nkabi ${ }^{1}$ and M.A. Meiring ${ }^{3,4}$}

${ }^{1}$ Paediatric Haematology Oncology, Department of Paediatrics, Steve Biko Academic Hospital, University of Pretoria, Pretoria, South Africa

${ }^{2}$ Haematology Oncology Service, Department of Paediatrics and Child Health, Red Cross War Memorial Children's Hospital, University of Cape Town, Cape Town, South Africa

${ }^{3}$ University of Cape Town, Cape Town, South Africa

${ }^{4}$ Paedspal, Cape Town, South Africa
Background: Palliative chemotherapy is used to stop or slow down the progression of cancer that is deemed to be incurable. In South Africa, delayed diagnosis of childhood cancer is a common problem for a variety of reasons, including lack of healthcare facilities, transport, information about the disease and delayed presentation because of traditional healer visits or other cultural issues. In these patients who present with advanced cancer, or in patients with relapsed cancer without realistic hope of cure, palliative chemotherapy can be offered in an attempt to improve the quality of life and prolong survival.

Objectives: This research study described the use of palliative chemotherapy in patients who received treatment at the Steve Biko Academic Hospital Paediatric Oncology Unit.

Method: A file review was of deceased patients who died in the period from January 2012 to December 2018 and who had received palliative chemotherapy as part of their cancer management performed. We reviewed diagnoses, palliative chemotherapy regimens, timing of initiation and stopping of palliative chemotherapy, and whether end of life decisions and discussions were documented.

Results: Of the 305 patient deaths recorded in the study period, a total of 74 patients had received palliative chemotherapy and were included in the file review. The most common diagnoses were neuroblastoma (18.2\%), retinoblastoma $(15.6 \%)$ and osteosarcoma $(14.3 \%)$. At the time of diagnosis, the median age of the patients was 6.0 years (range $0.3-17.6$ years). In 47 patients $(63.5 \%)$, the disease was deemed incurable at first diagnosis and palliative chemotherapy was given from the onset of treatment, while 27 patients $(36.5 \%)$ were given palliative chemotherapy at relapse of disease. The median time from last palliative chemotherapy to time of death was 30 days (range 0-742 days). The main documented aim of palliative chemotherapy was to improve symptom control (97.3\%), while parents' opinion played an important role in $32.9 \%$ of cases. About half of the patients $(41 / 74,55.4 \%)$ had documentation of symptomatic improvement.

Conclusion: This study not only provided a bird's-eye view on the use of palliative chemotherapy in children, but also showed clearly that the majority of decisions around end of life care and decision-making are better researched using a quantitative, prospective, interview-based approach. Repeated measurements of the child and family's quality of life and its associations with palliative chemotherapy should be a research priority in future, especially in our setting where many of the patients are from indigent communities and travel very far to reach oncology care.

\section{Life-world meets the biotechnical world: Patients' expectations and experiences of the computed tomography localisation event}

\section{G.T. Lovric}

University of Pretoria, Pretoria, South Africa 
Background: The radiation therapy (RT) localisation procedure is a transitional event between cancer diagnosis and radiation treatment in the cancer care continuum. The computed tomography (CT) localisation procedure is significant from an RT healthcare team perspective, where the technical aspects for radiation dose planning and the treatment delivery are prepared. To inform a patient-centred approach for effective care during this event, it is important to establish how patients' expectations and experiences are shaped at this point in the cancer journey.

Method: Ten patients with diverse demographics and diagnosis were interviewed immediately before and after undergoing the CT localisation procedure. The study was conducted using a qualitative research methodology with a phenomenological hermeneutic strategy to interpret and analyse the patients' reported expectations and experiences.

Results: In this presentation, an interpretation of the themes, sub-themes and categories that emerged from the analysis of the interviews will be offered. Although the aim of the study was focused on the expectations and experiences of the CT localisation event, other insights were gained. These were the experience of discovering the presence of the cancer and navigating the healthcare system to access treatment and the expectation for recovery that were significant enough for the patients to share during the interviews.

Conclusion: Although this study set out to provide insights to inform a patient-centred care at this juncture of the patients' journey, it also provides insights for person-centred care.

\section{Impact of various parameters on isodose coverage in the high dose-rate brachytherapy for cervical cancer}

\section{S. Acharya}

\section{HCG CCK Cancer Centre, Nairobi, Kenya}

Background: Cervical cancer is the most common cancer in Kenya, and high dose-rate (HDR) brachytherapy is an integral part of radiation treatment.

Objective: This retrospective study was designed to find the optimum inter-ovoid distance for best isodose coverage in cervical cancer HDR brachytherapy. The findings will help in situations where image-guided brachytherapy is not available or not feasible.

Methods: A retrospective analysis of 29 cases of uterine cervix randomly chosen in a 12-month period, from March 2018 to February 2019, was performed. Various parameters like ovoid separation, dose to bladder and rectum points as specified in ICRU-38 were recorded.

Results: All parameters recorded were correlated with the isodose coverage (AP and lateral) and the most significant parameter affecting the point $\mathrm{A}$ isodose distribution was inter-ovoid distance. The lateral isodose coverage increases with increase in ovoid separation up to approximately
$30 \mathrm{~mm}$, while the AP isodose coverage decreases significantly after $24 \mathrm{~mm}$ inter-ovoid distance.

Conclusion: Inter-ovoid distance of $20 \mathrm{~mm}-24 \mathrm{~mm}$ could be optimal considering both AP and lateral isodose coverages. This also translates to lesser chances of vaginal injury.

\section{Combining markers of hypermethylation with cytology or human papilloma virus-based cervical cancer screening in HIV-positive South African women}

\author{
W.W. Kremer ${ }^{1}$, E. Breytenbach ${ }^{2}$, C.J.L.M. Meijer ${ }^{1}$, \\ C. Visser $^{2}$ and G. Dreyer ${ }^{2}$ \\ ${ }^{1}$ Department of Pathology, UMC Vrije Universiteit, Amsterdam, \\ Netherlands \\ ${ }^{2}$ University of Pretoria, Pretoria, South Africa
}

Objectives: The aim of this study was to determine the role of molecular markers in cervical cancer screening strategies among South African women living with HIV (WLHIV).

Method: Data were collected from a South African cohort of WLHIV who also underwent cervical screening and colposcopy-directed biopsy. Data included cytology and HPV prevalence, HPV 16/18 genotyping and histology results. Detection of FAM19A4/miR124-2 hypermethylation was performed on DNA isolated from cervical scrapes. Diagnostic performance was determined of cytology and HPV tests, as single test strategies and in combination with FAM19A4/miR124-2 hypermethylation triage tests.

Results: A total of 285 women with valid results on all parameters were included in the analysis. Cytology provided the highest specificity (91.6\%) but lowest sensitivity (59.3\%), whereas a single HPV test provided the highest sensitivity (83.1\%) but lowest specificity $(66.4 \%)$. Combining cytology with methylation did not improve the performance compared with cytology alone: a slight increase in sensitivity was seen $(67.8 \%$ for cytology with methylation triage, and $62.7 \%$ for methylation with cytology triage) at the cost of a decrease in specificity (85.5\% and $87.2 \%)$. Triage of HPV-positive women with methylation increased specificity compared with the use of a single HPV test (76.1\%) or cytology while maintaining an acceptable sensitivity (72.9\%). Similar performance was observed for HPV16/18 with methylation triage (sensitivity $79.7 \%$, specificity $74.8 \%$ ). The number of women needed to refer to detect one CIN3+ case was lowest for cytology (1.5), but was only slightly higher for HPV with methylation triage or HPV16/18 with methylation triage (2.3 and 2.2, respectively).

Conclusion: Molecular screening strategies using HPV, with or without HPV16/18 genotyping, and FAM19A4/ miR124-2 hypermethylation have higher sensitivity, with an acceptable loss in specificity compared with current cytology screening practice. It can be feasible for detection of CIN3+ in South African WLHIV and further investigation is warranted. 


\section{Preliminary study on the design of a novel pelvic prosthesis spiral phantom for advanced radiotherapy verification}

\author{
K.E. Dumela ${ }^{1,2}$, I.T. Usman ${ }^{2}$ and O.M. Oderinde ${ }^{3}$ \\ ${ }^{1}$ Netcare Medical Physics CoE, Netcare Ltd., Johannesburg, \\ South Africa \\ ${ }^{2}$ University of the Witwatersrand, Johannesburg, South Africa \\ ${ }^{3}$ University of California San Diego, San Diego, CA, United States \\ of America
}

Background: Accurate radiotherapy dose verification is of great importance to optimise the local tumour control while sparing the organs at risk (OARs) and healthy tissues. In the presence of a high- $Z$ implanted material, the knowledge of planning and dosimetric verification are essential because of perturbation effect caused by the metal-tissue interface.

Objectives: The purpose of this study was to present the design and construction of a novel pelvic prosthesis spiral phantom for radiotherapy quality assurance (QA).

Method: The organ-specific pelvic prosthesis phantom consists of different removable inserts for ionisation chamber and spiral film. Ion chamber insert is suitable for cavity volume of $\leq 0.8 \mathrm{~cm}^{3}$, while film insert can accommodate a thin sheet and size of $3 \mathrm{~cm}$ and $20 \times 43 \mathrm{~cm}^{2}$, respectively. The 25-cm-thick dosimetric phantom also contains organsubstituted materials with densities of bladder, rectum, bony structure and prostate (cancer cell composition) embedded in water-equivalent nylon. One centimetre nylon is sandwiched between the titanium implant of $2.5 \mathrm{~cm}$ geometrical radius and the right hip bony structure for a tight grip. There will be a cylindrical hole crossing the prostate gland for placing the ionisation chamber and a spiral slot to enclose the film.

Results: This is a quick assemble phantom that is more convenient than liquid phantoms. The spiral phantom will be useful and flexible for dosimetric verification. It can determine the OAR doses for comprehensive treatment planning. The spiral shape of this phantom makes it suitable for arc radiotherapy QA.

\section{The impact of venous thromboembolism on the outcomes of patients with cervical carcinoma: A retrospective audit from January 2015 to December 2016}

\section{P.T. Goven Shiba and V. Sharma}

University of the Witwatersrand, Johannesburg, South Africa

Background: Venous thromboembolism (VTE) is a frequent cause of morbidity in patients with cervical cancer.

Objectives: The aim of this study was to investigate the outcomes of patients with cervical cancer and a deep vein thrombosis (DVT) in a South African population.

Methods: We retrospectively reviewed the records of cervical cancer patients who were admitted to our institution over a 2-year period from 2015 to 2016. Data collected included the age, stage, human immunodeficiency virus (HIV) status, proximity to radiotherapy and whether the course prescribed radiotherapy was completed. The survival of patients from diagnosis of VTE and the 2-year overall survival were evaluated using the Kaplan-Meier method. A univariate analysis was performed for age, clinical stage, HIV status, whether patients successfully completed radiotherapy and whether VTE was diagnosed before or after radiotherapy.

Results: We identified 47 patients with cervical cancer who were admitted to hospital with venous thromboembolism. The majority of patients (62\%) were stage IIIB. Of the patients, $60 \%$ were HIV-positive and $40 \%$ were HIV-negative. The median survival of patients from the time of diagnosis of VTE was 2.7 months (interquartile range [IQR]: 0.97-6.93 months) and the 12-month survival from diagnosis of VTE for this cohort was $17 \%$. Once a DVT was diagnosed, the survival becomes poor irrespective of age, stage, HIV status or whether the prescribed course of radiation was completed. The 2-year overall survival of this cohort was $29.8 \%$. The 2-year overall survival of patients who were diagnosed with a DVT before or during radiotherapy was significantly lower than that of patients who were diagnosed with DVT after radiotherapy $(12.5 \%$ vs. $38.7 \% ; p=0.001)$.

Conclusion: The diagnosis of VTE in patients with cervical cancer is an independent poor prognostic indicator and is associated with extremely poor survival. It is possible that poor prognosis reflects more aggressive tumour behaviour.

\section{Stereotactic radiosurgery for recurrent glioblastoma multiforme}

\section{T. Govender and T. Patrick}

Wits Donald Gordon Radiation Oncology Unit, Johannesburg, South Africa

Background: The most common malignant brain tumour in adults and most aggressive of all cancers is glioblastoma multiforme (GBM). Initial management of GBM is a combination of surgical resection, followed by fractionated radiotherapy and chemotherapy to improve overall survival. However, GBM recurs in majority of patients which is fatal. The utilisation of stereotactic radiosurgery (SRS) for the treatment of recurrent GBMs has been reported with variable success.

Objectives: The aim of this study was to evaluate the efficacy of SRS as a treatment for recurrent GBMs using a dose of $10 \mathrm{~Gy} \times 3$ fractions.

Method: Rates of radiation necrosis and neurological complications were performed.

Results: A dose of $10 \mathrm{~Gy} \times 3$ fractions using SRS after initial fractionated radiotherapy to $59.4 \mathrm{~Gy}$.

Conclusion: The results show that SRS is a relatively safe treatment option for recurrent GBM. 


\section{The use of high dose-rate endobronchial brachytherapy in rare adenoid cystic carcinoma tracheal recurrence: A case presentation}

\section{G.E. Paris ${ }^{1}$, F. Hendricks ${ }^{1}$, C.N.F. Koegelenberg ${ }^{2}$, B.W. Allwood ${ }^{2}$, M. Du Toit ${ }^{3}$ and M.A. Claasens ${ }^{3}$}

\author{
${ }^{1}$ Division of Radiation Oncology, Stellenbosch University, Cape \\ Town, South Africa \\ ${ }^{2}$ Division of Pulmonology, Stellenbosch University, Cape Town, \\ South Africa \\ ${ }^{3}$ Medical Imaging and Clinical Oncology, Tygerberg Hospital, \\ Tygerberg Campus, Cape Town, South Africa
}

Background: Adenoid cystic carcinoma is a rare tumour mostly occurring in the parotid gland, but can occur elsewhere in the aerodigestive and female genital tract. We present the case report of such a tumour in the lower trachea and detail the approach for its management.

Results: A 48-year-old white female presented to our department in 2017 with lower tracheal obstruction because of a primary adenoid cystic carcinoma. She underwent resection followed by high-dose external beam radiotherapy using the VMAT technique because of positive margins at multiple sites. Eighteen months later, she had an obvious symptomatic local recurrence for which high endobronchial therapy was successfully administered with a single dose of 10 Gy.

Conclusion: Endobronchial brachytherapy is feasible and safe in properly selected patients; the high single dose is desirable in a slow growing tumour such as the one in our patient. Similar cases will be too rare to generalise on its palliative effect, but observations on this patient should give some indication thereof; however, the extent of the tumour made complete inclusivity challenging.

\section{Radiophotoluminescence glass dosimetry end-to-end audit of high-dose rate gynaecological brachytherapy at Charlotte Maxeke Johannesburg Academic Hospital}

\section{M.Y. Lekganyane ${ }^{1}$, L. Ntombela ${ }^{1}$, V.N. Maselesele ${ }^{2}$ and M. Mathaoho ${ }^{1}$}

${ }^{1}$ Charlotte Maxeke Johannesburg Academic Hospital, Johannesburg, South Africa

${ }^{2}$ National Metrology Institute of South Africa, Pretoria, South Africa

Objectives: The aim of this study was to develop and perform the end-to-end for high-dose rate (HDR) brachytherapy for cervical cancer by comparing planned and delivered dose around a series of different gynaecological treatment applicators in water. This is done to improve the quality assurance in verifying the accuracy of the treatment system and to ensure that the brachytherapy software and hardware components are utilised properly and optimally for patient safety in brachytherapy treatments. One of the main objectives was to develop an HDR brachytherapy audit methodology.

Method: A phantom was designed using CAD software such that radio-photoluminescence glass (RPLG) dosimeters could be positioned accurately at point ' $A$ ', which is $2 \mathrm{~cm}$ parallel to the left and right side of the intrauterine (UI) tube and $2 \mathrm{~cm}$ superior to the surface of an ovoid or the cover of a ring applicator. Additional RPLG dosimeters were placed parallel to the dosimeter at point $A$ at $1 \mathrm{~cm}$ intervals up to $7 \mathrm{~cm}$ laterally. The phantom was designed in such a way that it could accommodate all the applicators that were used in this study. The designed water phantom was CT scanned with a 26-diameter ring and a 6-cm long IU. The scanned images were exported to the brachytherapy treatment planning system (Gammamed). The applicators were reconstructed on the images and plans were created to deliver 8 Gy to point A in water using the normal clinical protocol at Charlotte Maxeke Johannesburg Academic Hospital (CMJAH).

Results: The brachytherapy treatment planning system dose and the delivered dose differ by just more than $2 \%$. The combined standard uncertainties for each glass rod dosimeter were evaluated in this study, including the uncertainties from glass dosimeter characterisation.

Conclusion: The results show the possibility of establishing brachytherapy dosimetry audit using glass dosimeters. The audit will provide quality healthcare and physicists will fulfil legal requirements and minimise risk errors.

\section{The role of hyperthermia in radiotherapy}

\section{S. Moore and M. Klein}

Wits Donald Gordon Radiation Oncology Unit, Johannesburg, South Africa

Objectives: The aim of this study was to answer the following questions: (1) what is hyperthermia and how does it work? (2) Who stands to benefit from hyperthermia treatment? (3) What benefits does hyperthermia offer patients undergoing radiotherapy treatment?

Method: A hyperthermia unit was recently installed at the Wits Donald Gordon Medical Centre. We visited the unit and gained information from those directly involved in the treatment. Information was gathered from oncologists who refer their patients for hyperthermia treatment while receiving radiotherapy. We also collected information from studies conducted on the benefits of hyperthermia.

Results: The study highlighted a greater knowledge of hyperthermia and a better understanding of the selection process for patients. A clearer view of the advantages and disadvantages of the treatment was also provided.

Conclusion: Hyperthermia is a valuable complimentary treatment to radiation and could benefit all radiotherapy units.

\section{Volumetric modulated arc therapy for recurrent malignant synovial sarcoma}

\section{T. Nair, L. Ganas, B. Mofokeng and R. Hoosen}

Wits Donald Gordon Radiation Oncology Unit, Johannesburg, South Africa 
Background: Malignant synovioma is a rare form of cancer. It makes up about $10 \%$ of all sarcomas and usually occurs in the joints. Synovial sarcoma in the cardiac muscle is extremely uncommon. This case is about a 41-year-old woman who presented with shortness of breath and was eventually diagnosed with a synovial sarcoma of the cardiac muscle. The patient received a heart transplant and pacemaker in 2015 and later recurred in 2019 in the mediastinal nodes and both lungs.

Objectives: The aim of this study was to deliver 66 Gy in 33 treatments to the mediastinal nodes whilst limiting the dose to the surrounding organs at risk (OAR) (heart, liver, lungs and spinal cord) and pacemaker.

Method: The Eclipse ${ }^{\mathrm{TM}}$ Treatment Planning System was used to delineate the target volume, pacemaker and OARs. Both volumetric modulated arc therapy (VMAT) and a three-dimensional (3D) plan were created. These plans were then compared to assess the dose distribution in the target volume and the relevant doses to the critical structures and pacemaker.

Results: The VMAT plan was found to be more conformal with acceptable doses to the surrounding OAR as compared to the 3D plan.

Conclusion: The results show that VMAT produces a better outcome for the patient when treating with higher doses in the mediastinum.

\section{Correlation between PSA and bone scan findings in patients with prostate cancer at Grey's Hospital, KwaZulu-Natal: A retrospective chart review}

\section{F.B.M. Omar ${ }^{1,2}$, O. ElKoha ${ }^{1,2}$ and W. Sibanda ${ }^{3}$}

${ }^{1}$ Department of Radiotherapy and Oncology, Grey's Hospital, Pietermaritzburg, KwaZulu-Natal, South Africa

${ }^{2}$ University of KwaZulu-Natal, Durban, South Africa

${ }^{3}$ Biostatistics Unit, School of Nursing and Public Health, University of KwaZulu-Natal, Durban, South Africa

Objectives: The aim of this study was to investigate the correlation between PSA and bone scan findings in patients with prostate cancer at Grey's Hospital, Pietermaritzburg, KwaZulu-Natal, South Africa.

Methods: In this retrospective, randomised chart review study, 84 patients with prostate cancer were selected from patient files collected over a 3-year period, between 01 September 2015 and 30 September 2018, at Grey's Hospital in Pietermaritzburg, KwaZulu-Natal. Data were collected using Microsoft Excel. Data analysis was performed using IBM SPSS version 25 (Armonk, NY, USA) and SAS version 9.0 (SAS Institute, Cary, NC, USA). Continuous variables such as patient age were summarised using mean \pm standard deviation (s.d.), and medians (interquartile ranges [IQR]) for highly skewed data. Categorical variables such as age and race were summarised using proportions and percentages and compared using Pearson's chi-squared test or Fisher's exact test as deemed appropriate.
Results: Thirty-six (42.86\%) of the patients had both imageconfirmed bone metastasis and an elevated PSA level $(>8.6 \mathrm{ng} / \mathrm{mL}$ ), with a mean PSA level of $304.81 \pm 156$. Based on a chi-squared test, an increase in PSA level was found to be positively associated with an increased likelihood of positive bone scan findings $(p=0.012)$. Twenty-four $(92.31 \%)$ out of 26 patients with a PSA value of $\geq 100$ had a positive bone scan. No patient with serum PSA levels between $0.0 \mathrm{ng} / \mathrm{mL}$ and $4.0 \mathrm{ng} / \mathrm{mL}$ had a positive bone scan. Using the Fisher's exact test, no statistically significant association between patient age and incidence of bone metastasis was observed $(p=0.10)$. Bone metastasis had a statistically significant association with patients' race $(p=0.012)$, with black Africans having highest number of positive bone scans $(50.82 \%)$. The mean and median serum PSA concentration in patients with a positive bone scan was $560.54 \pm 890.93 \mathrm{ng} / \mathrm{mL}$ and 196.50 (IQR 56-802) ng/mL, respectively, whereas patients with a negative bone scan had a mean and median serum PSA level of $38.27 \pm 69.45 \mathrm{ng} / \mathrm{mL}$ and 20 (IQR 9-40.7) $\mathrm{ng} / \mathrm{mL}$, respectively. Using an unpaired $t$-test, the difference in mean serum PSA levels between patients with a positive bone scan and those without it was statistically significant $(p=0.0001)$. Only one patient $(1.19 \%)$ had a serum PSA value in the reference range $(0.0 \mathrm{ng} / \mathrm{mL}-4.0 \mathrm{ng} / \mathrm{mL})$. This patient's bone scan was reported to be normal.

Conclusion: Our study indicates that serum PSA levels have fairly high sensitivity $(100 \%)$ and positive predictive value $(43.37 \%)$ for bone metastasis. Out of 13 patients with PSA levels $\leq 10$, only $1(7.7 \%)$ had a positive bone scan finding. However, at PSA level $\leq 8.5 \mathrm{ng} / \mathrm{mL}, 0(0 \%)$ patient had a positive bone scan. The study also demonstrated that at PSA levels $\leq 24$, only $1(3.7 \%)$ patient out of 31 had a positive bone scan finding. It can be concluded that at PSA levels $\leq 24 \mathrm{ng} / \mathrm{mL}, 96.3 \%$ of the patients had a negative bone scan finding!

\section{Case study of a secondary meningioma in a previously irradiated field}

\section{G.E. Paris ${ }^{1}$, S. Lategaan ${ }^{2,3}$ and A. Maree ${ }^{2,3}$}

${ }^{1}$ Stellenbosch University, Cape Town, South Africa

${ }^{2}$ Tygerberg Hospital, Tygerberg Campus, Cape Town, South Africa ${ }^{3}$ Cape Peninsula University of Technology, Cape Town, South Africa

Background: Secondary malignancies following the treatment of childhood cancers are a rare but recognised complication. In the case of retinoblastoma, secondary osteosarcoma is more commonly noted; however, irrespective of the primary, radiation is a recognised risk factor for the development of malignant and benign meningioma.

Case presentation: We present a case study of a 43-year man who was treated curatively for retinoblastoma as an infant with multiple surgeries: radiation and chemotherapy. As an adult he developed an aggressive large meningioma in the treated area in the orbital socket and a second primary alongside the contralateral optic nerve. The approach to management of this is described and discussed as well as possible clinical scenarios associated with this occurrence. 
Conclusion: The development of this condition decades after his initial therapy presented unique challenges, despite that surgery and radiation therapy were possible.

\section{The advantages of using daily cone beam computed tomography for prostate and head and neck cancer patients}

\section{N.M. Tshishimbi ${ }^{1}$ and K.E. Dumela ${ }^{2}$ \\ ${ }^{1}$ Netcare Olivedale Hospital, Netcare Ltd., Randburg, South Africa ${ }^{2}$ Netcare Medical Physics CoE, Netcare Ltd., Johannesburg, South Africa}

Background: Advancement in radiotherapy techniques, like volumetric modulated arc therapy (VMAT), accurately shapes radiation dose to the tumour. This allows the delivery of higher radiation doses to targets while minimising the dose to organs at risks (OARs). These higher doses used in VMAT radiotherapy treatment delivery emphasise the need for treatment planning accuracy, reduced target delineation uncertainty and accurate patient setup. Volumetric imaging techniques, such as cone beam computed tomography (CBCT), has greatly improved the overall quality of VMAT treatment delivery by moving away from $2 \mathrm{D}$ verification of bony landmarks to the $3 \mathrm{D}$ positioning of the planning target volume (PTV) and OARs.

Objectives: The aim of this study was to measure the effect of daily CBCT verification imaging on treatment setup accuracy for prostate and head and neck $(\mathrm{H} / \mathrm{N})$ cancer patients treated with VMAT.

Method: The imaging study was performed on 30 patients with prostate cancer and 30 patients with $\mathrm{H} / \mathrm{N}$ cancer receiving 72 Gy and 60 Gy-66 Gy, respectively. Prostate patients receiving external beam radiotherapy post-brachytherapy were prescribed an additional 45 Gy. Treatment preparations for VMAT prostate patients included a full bladder and empty rectum protocol and $\mathrm{H} / \mathrm{N}$ cancer was all treated using a radiotherapy immobilisation mask. The daily verification process involved the acquisition of a CBCT image set followed by registration of the images with the planning $\mathrm{CT}$ performed during CT simulation at the beginning of the treatment course. Evaluation then included the determination of shifts in the longitudinal (Z-axis), vertical ( $Y$-axis) and lateral ( $X$-axis) directions aiming to position the patient in exactly the same position as originally planned. Corrections in $X-, Y$ - and Z-axes were made if necessary. The overall frequency of setup shifts was evaluated and the mean variations were calculated.

Results: Prostate mean variations are $1.6 \mathrm{~mm} \pm 1.0 \mathrm{~mm}$ along the $X$-axis, $2.0 \mathrm{~mm} \pm 1.0 \mathrm{~mm}$ along the $Y$-axis and $1.8 \mathrm{~mm} \pm$ $1.0 \mathrm{~mm}$ along the $Z$-axis, respectively. Head and neck mean variations are $1.5 \mathrm{~mm} \pm 0.5 \mathrm{~mm}$ along the $X$-axis, $1.5 \mathrm{~mm} \pm$ $0.5 \mathrm{~mm}$ along the $Y$-axis and $1.2 \mathrm{~mm} \pm 0.5 \mathrm{~mm}$ along the Z-axis, respectively.
Conclusion: Cone beam computed tomography has revolutionised and greatly improved the accuracy of pretreatment localisation information available to use for image guidance. This enables the treatment team to visualise the PTV and OAR prior to treatment delivery and allows the evaluation of setup accuracy and possible errors. This improves confidence that the dose distribution of the treatment plan is a fair representation of the actual PTV coverage delivered on the linear accelerator.

\section{Measured anisotropy and radial dose functions of the Oncoseed $6711 \mathrm{i}-125$ seed}

\section{C.J. Trauernicht ${ }^{1,2}$, E. Hering ${ }^{2}$, F. du Plessis ${ }^{3}$ and G. Maree ${ }^{4}$}

${ }^{1}$ Tygerberg Hospital, Stellenbosch University, Tygerberg Campus, Cape Town, South Africa

${ }^{2}$ University of Cape Town, Cape Town, South Africa

${ }^{3}$ University of the Free State, Bloemfontein, South Africa

${ }^{4}$ Groote Schuur Hospital, Cape Town, South Africa

Background: The anisotropy function of a brachytherapy seed accounts for the anisotropic dose distribution around the source. This function gives the angular variation of the dose rate about the source at each distance because of self-absorption, oblique filtration of photons through the seed capsule and scattering and absorption of photons in the medium. The radial dose function takes into account the effects of scatter and absorption in the medium along the transverse axis of the source, but it excludes any inverse square-type correction.

Methods: The anisotropy and radial dose functions were measured with thermoluminescent dosimeters (TLDs) and Gafchromic film in a specially designed solid water phantom. The anisotropy was measured to a distance of $5 \mathrm{~cm}$. The greyscale of the red colour channel of the Gafchromic film was converted to dose and a $1 \mathrm{~mm} \times 1 \mathrm{~mm}$ region of interest was drawn at each measurement point. Analysis was done using ImageJ. The anisotropy was measured with TLDs at distances of $2 \mathrm{~cm}, 3 \mathrm{~cm}$ and $5 \mathrm{~cm}$. Doses were measured at $0^{\circ}, \pm 30^{\circ}, \pm 60^{\circ}$ and $90^{\circ}$ at distances of $2 \mathrm{~cm}$ and $3 \mathrm{~cm}$, and every $10^{\circ}$ at a distance of $5 \mathrm{~cm}$. The TLD chip size was $3 \mathrm{~mm} \times 3 \mathrm{~mm} \times 0.9 \mathrm{~mm}$. The radial dose function was measured to a distance of $5 \mathrm{~cm}$ with film and with TLDs to a distance of $7 \mathrm{~cm}$.

Results: The anisotropy function and radial dose function measured with TLDs and Gafchromic film agreed with published data within measurement uncertainties.

Conclusion: The OncoSeed 6711 I-125 seed has been widely described in the literature. Measured data agree well with published data, but TLD data were closer to the published values than Gafchromic film data. 